\title{
Refined Reliability Combining for Binary Message Passing Decoding of Product Codes
}

\author{
Alireza Sheikh Member, IEEE, Alexandre Graell i Amat, Senior Member, IEEE, \\ Gianluigi Liva, Senior Member, IEEE, and Alex Alvarado Senior Member, IEEE.
}

\begin{abstract}
We propose a novel soft-aided iterative decoding algorithm for product codes (PCs). The proposed algorithm, named iterative bounded distance decoding with combined reliability (iBDD-CR), enhances the conventional iterative bounded distance decoding (iBDD) of PCs by exploiting some level of soft information. In particular, iBDD-CR can be seen as a modification of iBDD where the hard decisions of the row and column decoders are made based on a reliability estimate of the BDD outputs. The reliability estimates are derived using extrinsic message passing for generalized low-density-parity check (GLDPC) ensembles, which encompass PCs. We perform a density evolution analysis of iBDD-CR for transmission over the additive white Gaussian noise channel for the GLDPC ensemble. We consider both binary transmission and bit-interleaved coded modulation with quadrature amplitude modulation. We show that iBDD-CR achieves performance gains up to $0.51 \mathrm{~dB}$ compared to iBDD with the same internal decoder data flow. This makes the algorithm an attractive solution for very high-throughput applications such as fiber-optic communications.
\end{abstract}

Index Terms-Binary message passing, bounded distance decoding, complexity, high speed communications, hard decision decoding, product codes, quantization errors.

\section{INTRODUCTION}

The rediscovery of soft-decision (SD) iterative decoding algorithms and graph-based codes in the early 1990s allowed for the first time performance close to the theoretical limits with relatively low complexity. Turbo [1], and low-density paritycheck (LDPC) [2] codes were soon widely adopted in communications standards. However, the ever more demanding requirements in terms of throughput and power consumption, asks for new coding solutions. For example, current optical coherent transceivers operate at data rates of $400 \mathrm{Gbps}$ and the next frontier is 1 Tbps. Scaling conventional SD iterative decoders to such high throughputs is very challenging. This has spurred a great deal of research in the last few years on designing low-complexity coding and decoding schemes that can operate at very high throughputs while yet achieving performance close to that of conventional SD iterative schemes [3], [4].

A. Sheikh and A. Alvarado are with the Department of Electrical Engineering, Eindhoven University of Technology, PO Box 5135600 MB Eindhoven, The Netherlands (emails: \{asheikh, a.alvarado\}@tue.nl). The work of A. Sheikh and A. Alvarado has received funding from the European Research Council (ERC) under the European Union's Horizon 2020 research and innovation programme (grant agreement No 757791).

A. Graell i Amat is with the Department of Electrical Engineering, Chalmers University of Technology, SE-41296 Gothenburg, Sweden (email: alexandre.graell@chalmers.se).

G. Liva is with the Institute of Communications and Navigation of the German Aerospace Center (DLR), Münchner Strasse 20, 82234 Weßling, Germany (email: gianluigi.liva@dlr.de).
A key observation is that the main limitation to achieve very high throughputs arises from the high internal decoder data flow of SD decoders [5]. For this reason hard-decision (HD) decoders are appealing solutions when high throughputs are sought, as the internal decoder data flow can be kept reasonably small. For example, HD coding for optical communications is usually based on product-like codes with high rate Bose-Chaudhuri-Hocquenghem $(\mathrm{BCH})$ component codes, which can be efficiently decoded via bounded distance decoding (BDD) [5], [6]. The decoding is then performed based on BDD of the component codes and iterating between the row and column decoders, which we refer here to as iterative BDD (iBDD). The very high throughputs of HD product-like coding schemes, however, are achieved at the expense of a significant performance loss compared to their LDPC code counterparts, decoded via SD belief propagation.

Recently, several works have focused on the improvement of the performance of conventional iBDD of product-like codes targeting specifically very high throughputs [7]-[11]. The underlying idea in all these works is to generate some level of reliability information within the decoder to assist the conventional BDD. The result is an enhanced performance compared to iBDD while keeping the internal data flow low. Among the algorithms in [7], [8], [11], iBDD with scaled reliability (iBDD-SR) [8] is the one yielding the smallest increase in complexity, yet achieving about $0.3 \mathrm{~dB}$ performance improvement compared to iBDD for binary transmission. Following the principle introduced in [12], iBDD-SR is based on binary message passing (BMP) between component decoders and generates reliability information at the BDD output by scaling the decisions according to a reliability estimate of the decision. The reliability information, in the form of log-likelihood ratios is then added to the corresponding channel LLRs to form refined bit estimates (see [8, Fig. 2]). The decoding schemes in [9]-[11], [13], on the other hand, yield some additional coding gains but require the knowledge of the least reliable bits in the decoding process, and hence entail further complexity. Alternative constructions for high-throughput applications include coarsely-quantized low-density parity-check decoders [4], [12], [14], two-stage decoders [15], and SD-HD hybrid schemes based on concatenating a relatively weak SD code and an outer HD product-like code [16], [17].

This paper extends our previous work [8] in three different directions:

i. We derive a more accurate estimate of the reliability of the BDD outputs, which allows us to derive an improved combining rule for the BDD outputs and the 
channel LLRs. The resulting decoding algorithm, dubbed iterative bounded distance decoding with combined reliability (iBDD-CR) is shown to outperform iBDD-SR and, interestingly, also an idealized (genie-aided) iBDD that prevents miscorrections. The combining rule can be implemented by means of a small lookup table (LUT).

ii. We adapt the algorithm to bit interleaved coded modulation (BICM) with nonbinary modulation. For both binary and BICM transmission, we derive the density evolution (DE) equations for iBDD-CR, which provides an amenable analysis of the algorithm.

iii. We evaluate the effect of quantizing the channel LLRs and show that iBDD-CR has low sensitivity to quantization.

The combining rule derived in this work is based on DE analysis of (regular) generalized LDPC (GLDPC) codes ensembles that contain product codes (PCs) as ensemble members, under extrinsic message passing decoding. For the ensembles under consideration, the derived rule is optimal in the limit of large block lengths. Remarkably, the proposed combining rule provides a substantial coding gain with respect to iBDD-SR also when applied to the decoding of PCs, with gains that are consistent with the DE analysis findings. We show that iBDD-CR yields gains up to $0.51 \mathrm{~dB}$ compared to iBDD with an identical decoder data flow, making the algorithm particularly appealing for high-throughput applications.

Notation: We use boldface letters to denote vectors and matrices, e.g., $\boldsymbol{x}$ and $\boldsymbol{X}=\left[x_{i, j}\right]$, with $x_{i, j}$ representing the element corresponding to the $i$-th row and $j$-th column of $\boldsymbol{X}$. Moreover, $\boldsymbol{X}_{i,:}$ denotes the $i$-th row of $\boldsymbol{X}$. $|a|$ denotes the absolute value of $a,\lfloor a\rfloor$ the largest integer smaller than or equal to $a$, and $\lceil a\rceil$ the smallest integer larger than or equal to $a .(\cdot)^{\top}$ denotes the transpose operation. We also define $\hat{l} \in\{ \pm 1\}$ as the sign of value $l$. $\mathbb{R}$ is the set of real numbers and $p(\cdot)$ is the probability distribution or probability mass function of the continuous or discrete random variables (RVs), respectively. A Gaussian distribution with mean $\mu$ and variance $\sigma^{2}$ is denoted by $\mathcal{N}\left(\mu, \sigma^{2}\right)$. Furthermore, $\mathrm{G}\left(\lambda ; \mu, \sigma^{2}\right) \triangleq \frac{1}{\sqrt{2 \pi} \sigma} \exp \left(-\frac{(\lambda-\mu)^{2}}{2 \sigma^{2}}\right)$ stands for the Gaussian function with mean and variance $\mu$ and $\sigma^{2}$. We also denote by $\mathrm{Q}(x) \triangleq \frac{1}{\sqrt{2 \pi}} \int_{x}^{\infty} \mathrm{e}^{\frac{-\xi^{2}}{2}} \mathrm{~d} \xi$ the tail probability of the standard Gaussian distribution. The Hamming distance between vectors $\boldsymbol{a}$ and $\boldsymbol{b}$ is denoted by $\mathrm{d}_{\mathrm{H}}(\boldsymbol{a}, \boldsymbol{b})$. Finally, we define

$$
\overline{\mathbb{U}}(x)= \begin{cases}1 & \text { if } x<0 \\ 0 & \text { otherwise }\end{cases}
$$

\section{PRELIMINARIES}

We consider binary PCs with $\mathrm{BCH}$ component codes. Let $\mathcal{C}$ be an $(n, k) \mathrm{BCH}$ code with minimum Hamming distance $d_{\min }$ built over the Galois field $\operatorname{GF}\left(2^{v}\right)$ with (even) block length $n$ and information block length $k$ given by

$$
n=2^{v}-1, \quad k=2^{v}-v t-1,
$$

where $t \triangleq\left\lfloor\frac{d_{\text {min }}-1}{2}\right\rfloor$ is the error correcting capability of the code.
A (two-dimensional) PC with parameters $\left(n^{2}, k^{2}\right)$ and code rate $R=k^{2} / n^{2}$ is defined as the set of all $n \times n$ arrays such that each row and each column in the array is a codeword of $\mathcal{C}$. Accordingly, a codeword can be defined as a binary matrix $\boldsymbol{C}=\left[c_{i, j}\right]$. For ease of explanation, assume first transmission over the binary input additive white Gaussian noise (bi-AWGN) channel (in Sec. IV-B we also consider a bit-interleaved coded modulation (BICM)). The output of the bi-AWGN channel corresponding to code bit $c_{i, j}$ is thus given by

$$
y_{i, j}=x_{i, j}+n_{i, j},
$$

where $x_{i, j}=(-1)^{c_{i, j}}$ and $n_{i, j} \sim \mathcal{N}\left(0, \sigma^{2}\right)$. For a given $\mathrm{E}_{\mathrm{b}} / \mathrm{N}_{0}$ and code rate $R$, the noise variance is $\sigma^{2}=$ $\left(2 R \mathrm{E}_{\mathrm{b}} / \mathrm{N}_{0}\right)^{-1}$. The signal-to-noise ratio (SNR) per symbol is $\mathrm{E}_{\mathrm{s}} / \mathrm{N}_{0}=\frac{1}{2 \sigma^{2}}$. Let $\boldsymbol{L}=\left[l_{i, j}\right]$ be the matrix of channel LLRs and $\boldsymbol{R}=\left[r_{i, j}\right]$ the matrix of hard decisions at the output of the channel, i.e., $r_{i, j}$ is obtained taking the sign of $l_{i, j}$ and mapping $-1 \mapsto 1$ and $+1 \mapsto 0$. We denote this mapping by $\mathrm{B}(\cdot)$, i.e., $r_{i, j}=\mathrm{B}\left(l_{i, j}\right)$. With some abuse of notation we will write $\boldsymbol{R}=\mathrm{B}(\boldsymbol{L})$.

PCs are conventionally decoded using BDD of the component codes. Here we briefly explain BDD. Consider the decoding of an arbitrary row component, which is an $1 \times n$ array. Specifically, assume decoding of the transmitted component codeword $\boldsymbol{c}=\left(c_{1}, \ldots, c_{n}\right)$ based on the hard-detected bits at the channel output, $\boldsymbol{r}=\left(r_{1}, \ldots, r_{n}\right)$. BDD corrects all error patterns with Hamming weight up to the error-correcting capability of the code $t$. If the weight of the error pattern is larger than $t$ and there exists another codeword $\tilde{\boldsymbol{c}} \in \mathcal{C}$ with $\mathrm{d}_{\mathrm{H}}(\tilde{\boldsymbol{c}}, \boldsymbol{r}) \leq t$, then BDD erroneously decodes $\boldsymbol{r}$ onto $\tilde{\boldsymbol{c}}$ and a so-called miscorrection occurs. Otherwise, if such a codeword does not exist, BDD fails. In this case, the bounded distance (BD) decoder may output its input $\boldsymbol{r}$, or it may declare a decoding failure (often referred to as an erasure). The decoding of PCs can be accomplished in an iterative fashion based on BDD of the component codes and iterating between the row and column decoders, which we refer to as iBDD. In the case of iBDD, if a local BD decoder fails, it outputs the input vector.

\section{Iterative Bounded Distance Decoding With COMBINED RELIABILITY}

Recently, a variant of iBDD called iBDD-SR has been proposed, where the BDD outbound messages are modified based on the channel reliabilities to reduce miscorrections. A detailed explanation of iBDD-SR can be found in [8]. We highlight that iBDD-SR exploits an estimate of the reliability of the BDD decisions to produce weighted sums of BDD outputs and channel LLRs (see [8, Fig. 2]). We provide next an improved combining rule, based on an enhanced model of the component code decoder behavior. The new rule will be shown to be superior to the weighted sum approach of [8], in terms of both asymptotic decoding threshold and finite-length performance.

Consider the decoding of an $\left(n^{2}, k^{2}\right)$ PC and let $\Psi^{\mathrm{c},(\ell-1)}=$ $\left[\psi_{i, j}^{\mathrm{c},(\ell-1)}\right]$ be the decoding result of the $n$ column codes at 


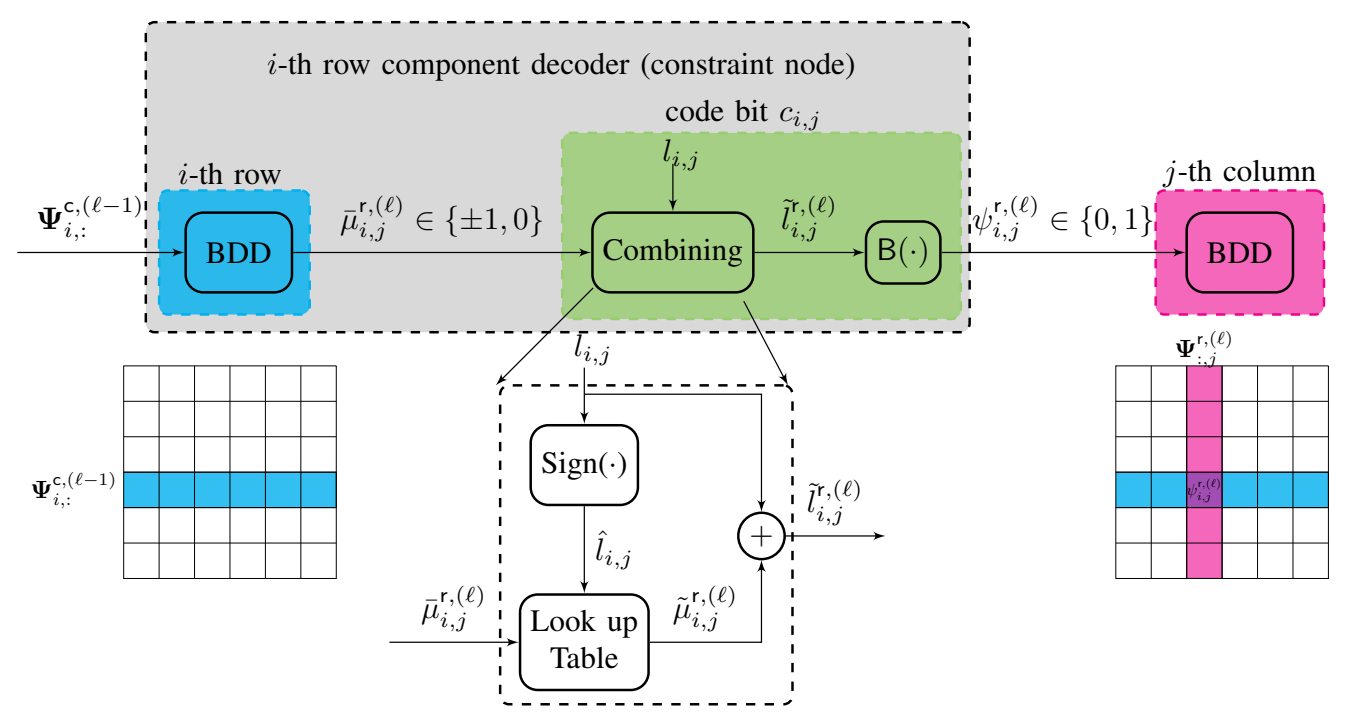

Fig. 1: Block diagram illustrating the message passing in iBDD-CR corresponding to $i$ th row decoding at iteration $\ell$. The diagram shows the decision on code bit $c_{i, j} . \mathbf{\Psi}_{i,:}^{\mathrm{c},(\ell-1)}$ is the input to BDD, $\bar{\mu}_{i, j}^{\mathrm{r},(\ell)}$ is the output of BDD, $l_{i, j}$ is the channel LLR, $\hat{l}_{i, j}$ is sign of channel LLR, $\tilde{\mu}_{i, j}^{\mathrm{r},(\ell)}$ is the output of LUT given in Table $\mathrm{I}$

iteration $\ell-1$, where $\psi_{i, j}^{\mathrm{c},(\ell-1)}$ corresponds to the decision on code bit $c_{i, j}$. The input of the row decoders at iteration $\ell$ is $\Psi^{\mathrm{c},(\ell-1)}$. Without loss of generality, we assume the decoding of the $i$ th row code at iteration $\ell$, hence, the input of the decoder is $\Psi_{i:}^{\mathrm{c},(\ell-1)} 1$ The block diagram of the iBDD-CR algorithm corresponding to the $i$ th row decoding at iteration $\ell$ is schematized in Fig. 1 . Let $\bar{\mu}_{i, j}^{r}$ denote the output of the $i$ th row BD decoder corresponding to code bit $c_{i, j} \cdot \bar{\mu}_{i, j}^{r}$ takes values on a ternary alphabet, $\bar{\mu}_{i, j}^{r} \in\{ \pm 1,0\}$, where the decoded bits are mapped according to $0 \mapsto+1$ and $1 \mapsto-1$ if BDD is successful, and the output is 0 in the case of a decoding failure. Let $\tilde{l}_{i, j}^{\text {ro }}(\ell)$ be the soft value of code bit $c_{i, j}$ at iteration $\ell$, which is formed based on the values of the BDD output and the channel LLRs, i.e., $\bar{\mu}_{i, j}^{r,(\ell)}$ and $l_{i, j}$, respectively. In Sec. IV, we derive a closed-form expression for $\tilde{l}_{i, j}^{\text {r, }(\ell)}$ via a DE analysis ${ }^{2}$ For now, assume that combining $\bar{\mu}_{i, j}^{\mathrm{r},(\ell)}$ and $l_{i, j}$ results in a soft value $\tilde{l}_{i, j}^{r,(\ell)}$. Then, the hard decision on the code bit $c_{i, j}$ produced by the $i$ th row decoder is formed as

$$
\psi_{i, j}^{\mathrm{r},(\ell)}=\mathrm{B}\left(\tilde{l}_{i, j}^{\mathrm{r},(\ell)}\right),
$$

where ties can be broken with any policy (see Sec. II for the definition of $B(\cdot)$ ).

The hard decision $\psi_{i, j}^{r,(\ell)}$ is the message passed from the $i$-th row decoder to the $j$-th column decoder. In particular, after applying this procedure to all row decoders, the matrix $\Psi^{\mathbf{r},(\ell)}=\left[\psi_{i, j}^{\mathbf{r},(\ell)}\right]$ is formed and used as the input for the $n$ column decoders, and column decoding based on $\boldsymbol{\Psi}^{r,(\ell)}$ is performed. Assume the decoding of the $j$ th column code at iteration $\ell$, hence, the input of the decoder is $\Psi_{:, j}^{r,(\ell)}$. As before, we assume that the output of the $j$ th column BD decoder

\footnotetext{
${ }^{1}$ Recall our notation where $\boldsymbol{X}_{i, \text { : }}$ denotes the $i$-th row of $\boldsymbol{X}$.

${ }^{2}$ Note that the expression is derived under the assumption of an asymptotically-large block length, under an extrinsic message passing variation of the algorithm, as will be discussed in Sec. IV
}

corresponding to code bit $c_{i, j}$, denoted by $\bar{\mu}_{i, j}^{\mathrm{c}}$, takes values on $\{ \pm 1,0\}$. Similar to row decoding, $\tilde{l}_{i, j}^{c,(\ell)}$ is formed based on combining $\bar{\mu}_{i, j}^{\mathrm{c}}$ and $l_{i, j}$. Then, the hard decision on code bit $c_{i, j}$ produced by the $j$ th column decoder is formed as

$$
\psi_{i, j}^{\mathrm{c},(\ell)}=\mathrm{B}\left(\tilde{l}_{i, j}^{\mathrm{c},(\ell)}\right) .
$$

The matrix $\Psi^{\mathrm{c},(\ell)}=\left[\psi_{i, j}^{\mathrm{c},(\ell)}\right]$ is passed to the $n$ row decoders for the decoding iteration $\ell+1$. The iterative process continues until a maximum number of iterations is reached.

With reference to Fig. 1, observe that in the proposed algorithm the component code decoders exchange only (binary) hard decisions. Hence, the contribution of the messages passed among component decoders to the overall internal decoder data flow is comparable to that of conventional iBDD [5 Sec. III.A].

\section{Density Evolution AnAlysis of IBDD-CR FOR GLDPC CODE ENSEMBLES}

\section{A. Density Evolution Analysis for the bi-AWGN Channel}

We provide in this section a DE analysis of PCs, under iBDD-CR decoding. More specifically, we analyze PCs as members of regular GLDPC code ensembles [18]. To do so, we first recall the Tanner graph representation of a PC [19]. A two-dimensional PC defined by an $(n, k)$ component code (used for both rows and columns of the codeword array) can be represented by a graph consisting of two sets of nodes: $n^{2}$ degree- 2 variable nodes (VNs) and $2 n$ degree- $n$ constraint nodes (CNs). Each VN is associated to a codeword bit, and each $\mathrm{CN}$ is associated to a row/column code. VNs and CNs are then connected by edges according to the constraints defined by the PC construction. An example is provided in Fig. 2 for the case of $n=3$ component code.

PCs can be seen as a special class of GLDPC codes for which the connections between VNs and CNs are directly 


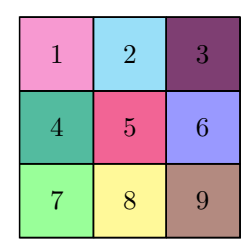

(a)

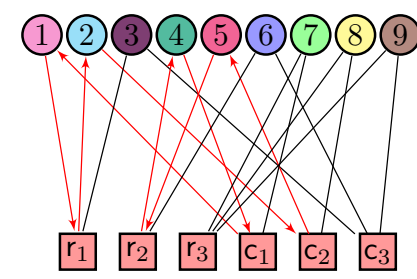

(b)

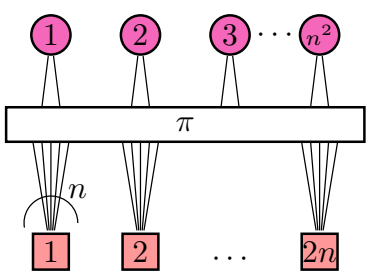

(c)

Fig. 2: (a) The PC code array with 9 bits. (b) Tanner graph of the PC. $r_{1}, r_{2}$, and $r_{3}\left(c_{1}, c_{2}\right.$, and $c_{3}$ ) stand for the first, second, and third BCH row (column) constraint nodes. A length-8 cycle is highlighted in the Tanner graph. (c) Tanner graph of a generic regular GLDPC code with $n^{2}$ degree-2 VNs and $2 n$ degree- $n$ CNs.

defined by the PC structure. The deterministic nature of the Tanner graph of PCs has a major consequence in terms of DE analysis. Indeed, DE can applied to graphs whose nodes have a tree-like neighborhood down to a certain depth (which is directly related to the number of decoding iterations). To determine the limiting behavior in terms of iterative decoding threshold, the number of iterations (and, consequently, the depth for which a tree-like neighborhood is required) has to be taken to infinity. This demands for the block length to grow large (i.e., in the limit, to infinity). However, even if one would be able to construct a sequence of PCs with increasing block length, the requirement of a tree-like neighborhood down to a growing depth cannot be attained by any PC. The girth of the Tanner graph of a PC is, in fact, 8 (a length- 8 cycle in the Tanner graph of the PC of Fig. 2(b) is highlighted), jeopardizing the possibility to perform a proper $\mathrm{DE}$ analysis ${ }^{3}$ Hence, rather than analyzing a specific $\mathrm{PC}$, we resort to the analysis of the GLDPC ensemble encompassing the PC.

Fig. 2(c) depicts the Tanner graph of a generic PC with $n^{2}$ degree- 2 VNs and $2 n$ degree- $n$ CNs, where the permutation of the edges is represented by the edge interleaver $\pi$. The PC is defined by a particular permutation. Note also that this Tanner graph corresponds to that of a regular GLDPC code for which all CNs are associated the same $(n, k)$ component code. The set of codes defined by all possible edge permutations yields the GLDPC code ensemble, which contains among its members the two-dimensional PC based on the $(n, k)$ component code. To perform a DE analysis, we will need to untangle the number of VNs and $\mathrm{CNs}$ from the component code block length: The regular GLDPC code ensembles on interest will be hence defined by the CNs component code, the degree of the VNs (which is 2), and the block length (i.e., the number of VNs).

Remark 1: The Tanner graph of a product/GLDPC code can be used to describe the message-passing schedule between VNs and CNs. Typically, GLDPC ensembles are analyzed under the assumption of a flooding schedule, i.e., at each iteration VNs pass a message to all the neighboring $\mathrm{CNs}$, and all CNs pass a message to their neighboring VNs. While the principle can be applied to PCs, too, in the following we will analyze a message passing schedule that follows from

\footnotetext{
${ }^{3}$ A notable exception, where a DE analysis of PCs can be performed, was described in [20] for the binary erasure channel for the limiting case where the code rate tends to 1 .
}

the decoding algorithm described in Sec. III. To this aim, we divide the CNs into two classes (or types): The class of $\mathrm{CNs}$ associated to row decoders and the class of $\mathrm{CNs}$ associated to column decoders. The decoding schedule, hence, will involve two half-iterations: In the first half-iteration, only $\mathrm{CNs}$ associated to column decoders are active, while in the second half-iteration only $\mathrm{CNs}$ associated to row decoders are active.

Remark 2: A further difference in the analysis with respect to the classical analysis of GLDPC code ensembles stems from the DE analysis approach proposed in [6] for GLDPC code ensembles under iBDD. Typically, a VN in a GLDPC code Tanner graph gets as input the corresponding codeword bit channel observation, and it forwards to the neighboring CNs an updated belief. The updated belief accounts for the channel observation and the extrinsic information provided at the previous iteration, by the CNs output. As suggested in [6], when analyzing the iBDD algorithm it is particularly convenient to provide the channel observations as input to each $\mathrm{CN}$ directly, and to let VNs act as simple message routers (i.e., they forward the message received along one edge over the other edge without performing any modification). We adopt this approach in the following. It follows that the combining of the channel soft information with the output of the local $\mathrm{BD}$ decoders takes place within the $\mathrm{CNs}$, as emphasized in Fig. 1

In order to proceed with the $\mathrm{DE}$ analysis, a further important aspect needs to be addressed. In particular, the decoding rule specified for the component code decoders in Sec. III has to be modified in order to render the message passing extrinsic. From (3)-44, one can infer that the standard row (column) decoding of PCs using iBDD-CR does not fall into the extrinsic decision rule category, as the input of row (column) decoder for iteration $\ell$ is the output of column (row) decoder from the iteration $\ell-1$. Therefore, for analysis purposes, similar to [6], [8] we modify the algorithm such that the BDD of the component code is substituted by an extrinsic rule relying on BDD [6, Sec. II.B].

The iBDD-CR decoding algorithm with extrinsic BDD of the component codes is explained in the following. Without loss of generality, we consider the decoding of the $i$-th row of the PC at iteration $\ell$, corresponding to the transmitted component code codeword $\boldsymbol{c}=\left(c_{1}, \ldots, c_{n}\right)$, where the input for the $i$-th row decoder is $\Psi_{i,:}^{\mathrm{c},(\ell-1)}=\left(\psi_{i, 1}^{\mathrm{c},(\ell-1)}, \ldots, \psi_{i, n}^{\mathrm{c},(\ell-1)}\right)$. 
For the decision on the code bit $c_{i, j}$, the $j$-th component of $\boldsymbol{\Psi}_{i,:}^{\mathrm{c},(\ell-1)}$ is substituted by the channel output $r_{i, j}$, and hence, $\tilde{\psi} \triangleq\left(\psi_{i, 1}^{\mathrm{c},(\ell-1)}, \ldots, r_{i, j}, \ldots, \psi_{i, n}^{\mathrm{c},(\ell-1)}\right)$ is used as the input for BDD. After performing extrinsic BDD, the outbound message on code bit $c_{i, j}\left(\bar{\mu}_{i, j}^{\mathrm{r}}\right)$ is

$$
\bar{\mu}_{i, j}^{\mathrm{r}}= \begin{cases}(-1)^{c_{i}} & \text { if } \mathrm{d}_{\mathrm{H}}(\boldsymbol{c}, \tilde{\boldsymbol{\psi}}) \leq t \\ (-1)^{\tilde{c}_{i}} & \text { if } \mathrm{d}_{\mathrm{H}}(\boldsymbol{c}, \tilde{\boldsymbol{\psi}})>t \text { and } \exists \tilde{\boldsymbol{c}} \text { such that } \mathrm{d}_{\mathrm{H}}(\tilde{\boldsymbol{c}}, \tilde{\boldsymbol{\psi}}) \leq t \\ 0 & \text { otherwise }\end{cases}
$$

where $\tilde{\boldsymbol{c}}=\left(\tilde{c}_{1}, \ldots, \tilde{c}_{n}\right)$ is a valid component codeword. The same decoding rule can be employed for iBDD-CR decoding with extrinsic BDD of column codes.

Remark 3: The extrinsic decoding rule requires decoding $n$ times each component code, which is complex for a practical system. We highlight that we only use the extrinsic decoding rule to derive the $\mathrm{DE}$ analysis, whereas for the performance evaluation via simulations we use the more practical decoder outlined in Sec. III i.e., the standard (intrinsic) row/column decoding of the component codes is employed.

We consider transmission over the bi-AWGN channel where a length- $n \mathrm{BCH}$ component code with error-correcting capability $t$ is assumed at the CNs. We denote by $p_{\text {ch }}$ the channel output error probability yielded by applying hard detection to the bi-AWGN channel output, i.e., $p_{\text {ch }}=\mathrm{Q}\left(\frac{1}{\sigma}\right)$. We consider two sets of equal size for $\mathrm{CNs}$, where each set defines a $\mathrm{CN}$ type. We refer to the two $\mathrm{CN}$ types as row and column $\mathrm{CN}$ types. Each $\mathrm{VN}$ is connected to one row-type $\mathrm{CN}$ and to one column-type $\mathrm{CN}$. Each decoding iteration consists of one row $\mathrm{CN}$ processing, followed by one column $\mathrm{CN}$ processing. In the following, we denote by $x$ the error probability associated to the messages exchanged by the component decoders (via VNs). In particular, we denote by $x^{\mathrm{r},(\ell)}$ and $x^{\mathrm{c},(\ell)}$ the message error probability at the output of the row component decoder (row-type $\mathrm{CN}$ ) and column component decoder (column-type $\mathrm{CN}$ ), respectively, at the $\ell$ th iteration. The message error probability at the input of a row-type $\mathrm{CN}$ at the $\ell$ th iteration is given by $x^{c,(\ell-1)}$, whereas the message error probability at the input of a column-type CN during the $\ell$ th iteration is $x^{\mathrm{r},(\ell)}$.

Without loss of generality, consider the row-type $\mathrm{CN}$ operation at iteration $\ell$ of DE.The combining yields a soft estimate for code bit $c_{i, j}$, i.e., $\tilde{l}_{i, j}^{r,(\ell)}$, given the corresponding BDD output and channel LLR are $\bar{\mu}_{i, j}^{\mathrm{r},(\ell)}$ and $l_{i, j}$, respectively. By applying Bayes' rule to the definition of $\tilde{l}_{i, j}^{r,(\ell)}$, we have

$$
\begin{aligned}
\tilde{l}_{i, j}^{r,(\ell)} \triangleq & \ln \frac{p\left(\bar{\mu}_{i, j}^{r,(\ell)}, l_{i, j} \mid c_{i, j}=0\right)}{p\left(\bar{\mu}_{i, j}^{r,(\ell)}, l_{i, j} \mid c_{i, j}=1\right)} \\
= & \ln \frac{p\left(\bar{\mu}_{i, j}^{r,(\ell)} \mid l_{i, j}, c_{i, j}=0\right)}{p\left(\bar{\mu}_{i, j}^{r,(\ell)} \mid l_{i, j}, c_{i, j}=1\right)}+\ln \frac{p\left(l_{i, j} \mid c_{i, j}=0\right)}{p\left(l_{i, j} \mid c_{i, j}=1\right)} \\
= & \ln \frac{p\left(\bar{\mu}_{i, j}^{r,(\ell)} \mid l_{i, j}, c_{i, j}=0\right)}{p\left(\bar{\mu}_{i, j}^{r,(\ell)} \mid l_{i, j}, c_{i, j}=1\right)}+l_{i, j} .
\end{aligned}
$$

where $l_{i, j}=\frac{2}{\sigma^{2}} y_{i, j}$. It follows $l_{i, j} \sim \mathcal{N}\left(2 / \sigma^{2}, 4 / \sigma^{2}\right)$ if $c_{i, j}=$ 0 and $l_{i, j} \sim \mathcal{N}\left(-2 / \sigma^{2}, 4 / \sigma^{2}\right)$ if $c_{i, j}=1$.
In general, computing the first term of (6) is a formidable task, since the extrinsic decoding rule (5) results in a statistical dependence between $\bar{\mu}_{i, j}^{\mathrm{r},(\ell)}$ and $l_{i, j}$. The trick for computing this term is that $\bar{\mu}_{i, j}^{r,(\ell)}$ only depends on the hard value $\hat{l}_{i, j}$ and not on the reliability $\left|l_{i, j}\right|^{4}$ In fact, the extrinsic decoding rule imposes that $l_{i, j} \rightarrow \hat{l}_{i, j} \rightarrow \bar{\mu}_{i, j}^{r,(\ell)}$ forms a Markov chain, i.e., given $\hat{l}_{i, j}, \bar{\mu}_{i, j}^{\mathrm{r},(\ell)}$ and $l_{i, j}$ are statistically independent. Therefore, one can re-state (6) as

$$
\tilde{l}_{i, j}^{r,(\ell)}=\tilde{\mu}_{i, j}^{r,(\ell)}+l_{i, j}
$$

where $\tilde{\mu}_{i, j}^{\mathrm{r},(\ell)}$ is defined as

$$
\tilde{\mu}_{i, j}^{\mathrm{r},(\ell)} \triangleq \ln \frac{p\left(\bar{\mu}_{i, j}^{\mathrm{r},(\ell)} \mid \hat{l}_{i, j}, c_{i, j}=0\right)}{p\left(\bar{\mu}_{i, j}^{\mathrm{r},(\ell)} \mid \hat{l}_{i, j}, c_{i, j}=1\right)} .
$$

Similarly, for the column-type CN operation the soft value of the $\mathrm{VN}$ corresponding to code bit $c_{i, j}$ is derived as

$$
\tilde{l}_{i, j}^{\mathrm{c},(\ell)}=\tilde{\mu}_{i, j}^{\mathrm{c},(\ell)}+l_{i, j}
$$

where $\tilde{\mu}_{i, j}^{\mathrm{c},(\ell)}$ is defined as

$$
\tilde{\mu}_{i, j}^{\mathrm{c},(\ell)} \triangleq \ln \frac{p\left(\bar{\mu}_{i, j}^{\mathrm{c},(\ell)} \mid \hat{l}_{i, j}, c_{i, j}=0\right)}{p\left(\bar{\mu}_{i, j}^{\mathrm{c},(\ell)} \mid \hat{l}_{i, j}, c_{i, j}=1\right)} .
$$

The derivation of $\tilde{\mu}_{i, j}^{\mathrm{r},(\ell)}$ and $\tilde{\mu}_{i, j}^{\mathrm{c},(\ell)}$ under extrinsic iBDD-CR decoding is provided by the next proposition.

Proposition 1: Over the bi-AWGN channel, the values of $\tilde{\mu}_{i, j}^{\mathrm{r},(\ell)}$ and $\tilde{\mu}_{i, j}^{\mathrm{c},(\ell)}$ are provided in Table $\mathrm{I}$ and Table II respectively, where the message error probability at the rowtype and column-type $\mathrm{CN}$ at the $\ell$ th iteration is given by 11 and (12), respectively, with $x^{c,(0)}=p_{\mathrm{ch}}$, and the values of $f^{P^{\mathrm{e}}}(x), f^{P^{c}}(x), f^{Q^{\mathrm{e}}}(x), f^{Q^{c}}(x), f^{P^{\epsilon}}(x), f^{Q^{\epsilon}}(x)$ in Tables III are derived in 20]-25].

The proof of Proposition 1 is given in Appendix $\mathrm{A}$. Owing to (6), we remark that the combining rule is optimal for GLDPC code ensembles in the sense of minimizing the message error probability, under extrinsic message passing decoding, in the limit of infinitely large blocks.

\section{B. Density Evolution Analysis for BICM with $M^{2}-Q A M$}

BICM [21], [22] (see Fig. 3) has become a de-facto standard in optical communications [23], [24] due to the inherent flexibility and implementation simplicity. One can see the $M^{2}$ QAM modulation as a Cartesian product of two amplitude shift keying (ASK) modulations, i.e, the real and imaginary parts of each $M^{2}$-QAM symbol are chosen form an $M$-ASK constellation. More specifically, for $M=2^{m}$, the constellation points for both real and imaginary parts of the $M^{2}$-QAM symbol are chosen from $\mathcal{X} \triangleq\left\{\left(-2^{m}+1\right) \cdot \Delta, \ldots,-\Delta, \Delta, \ldots,\left(2^{m}-1\right) \cdot \Delta\right\}$ where $\Delta=\sqrt{\frac{3}{2\left(M^{2}-1\right)}}$ is a scaling factor which normalizes

\footnotetext{
${ }^{4}$ Recall that, for what concerns the computation of $\tilde{l}_{i, j}^{r,(\ell)}$ in the extrinsic message passing decoding rule, the input of the row decoder corresponding to code bit $c_{i, j}$ is given by $r_{i, j}=\mathrm{B}\left(l_{i, j}\right)$. As $\mathrm{B}(\cdot)$ is a mapper operating on the sign of its input, $\bar{\mu}_{i, j}^{\mathrm{r},(\ell)}$ only depends on $\hat{l}_{i, j}$.
} 


$$
\begin{aligned}
& x^{\mathrm{r},(\ell)}=f^{P^{\mathrm{e}}}\left(x^{\mathrm{c},(\ell-1)}\right) \mathrm{Q}\left(\frac{\sigma}{2} \min \left(\ln \left(\frac{f^{P^{\mathrm{e}}}\left(x^{\mathrm{c},(\ell-1)}\right)}{f^{Q^{\mathrm{c}}}\left(x^{\mathrm{c},(\ell-1)}\right)}\right), 0\right)+\frac{1}{\sigma}\right)+f^{P^{\mathrm{c}}}\left(x^{\mathrm{c},(\ell-1)}\right) \mathrm{Q}\left(\frac{\sigma}{2} \min \left(\ln \left(\frac{f^{P^{\mathrm{c}}\left(x^{\mathrm{c},(\ell-1)}\right)}}{f^{Q^{\mathrm{e}}}\left(x^{\mathrm{c},(\ell-1)}\right)}\right), 0\right)+\frac{1}{\sigma}\right)+
\end{aligned}
$$

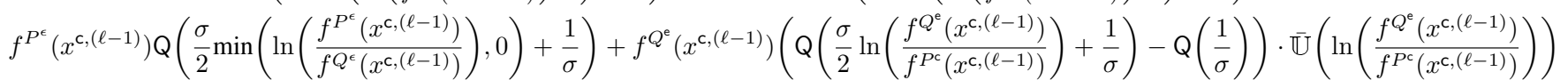

$$
\begin{aligned}
& +f^{Q^{\mathrm{c}}}\left(x^{\mathrm{c},(\ell-1)}\right)\left(\mathrm{Q}\left(\frac{\sigma}{2} \ln \left(\frac{f^{Q^{\mathrm{c}}}\left(x^{\mathrm{c},(\ell-1)}\right)}{f^{P^{\mathrm{e}}}\left(x^{\mathrm{c},(\ell-1)}\right)}\right)+\frac{1}{\sigma}\right)-\mathrm{Q}\left(\frac{1}{\sigma}\right)\right) \cdot \overline{\mathbb{U}}\left(\ln \left(\frac{f^{Q^{\mathrm{c}}}\left(x^{\mathrm{c},(\ell-1)}\right)}{f^{P^{\mathrm{e}}}\left(x^{\mathrm{c},(\ell-1)}\right)}\right)\right) \\
& +f^{Q^{\epsilon}}\left(x^{c,(\ell-1)}\right)\left(\mathrm{Q}\left(\frac{\sigma}{2} \ln \left(\frac{f^{Q^{\epsilon}}\left(x^{c,(\ell-1)}\right)}{f^{P^{\epsilon}}\left(x^{c,(\ell-1)}\right)}\right)+\frac{1}{\sigma}\right)-\mathrm{Q}\left(\frac{1}{\sigma}\right)\right) \cdot \overline{\mathbb{U}}\left(\ln \left(\frac{f^{Q^{\epsilon}}\left(x^{c,(\ell-1)}\right)}{f^{P^{\epsilon}}\left(x^{c,(\ell-1)}\right)}\right)\right)
\end{aligned}
$$

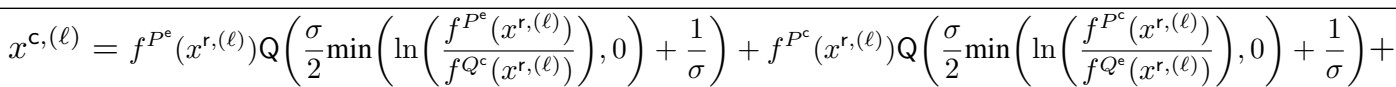

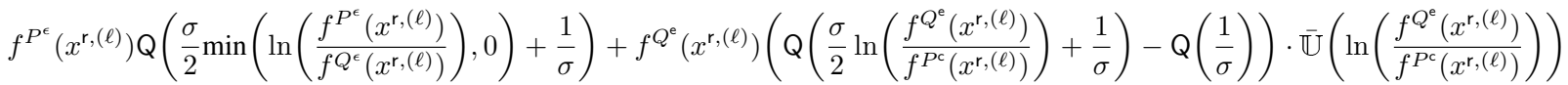

$$
\begin{aligned}
& +f^{Q^{c}}\left(x^{\mathrm{r},(\ell)}\right)\left(\mathrm{Q}\left(\frac{\sigma}{2} \ln \left(\frac{f^{Q^{\mathrm{c}}}\left(x^{\mathrm{r},(\ell)}\right)}{f^{P^{\mathrm{e}}}\left(x^{\mathrm{r},(\ell)}\right)}\right)+\frac{1}{\sigma}\right)-\mathrm{Q}\left(\frac{1}{\sigma}\right)\right) \cdot \overline{\mathbb{U}}\left(\ln \left(\frac{f^{Q^{\mathrm{c}}}\left(x^{\mathrm{r},(\ell)}\right)}{f^{P^{e}}\left(x^{\mathrm{r},(\ell)}\right)}\right)\right) \\
& +f^{Q^{\epsilon}}\left(x^{\mathrm{r},(\ell)}\right)\left(\mathrm{Q}\left(\frac{\sigma}{2} \ln \left(\frac{f^{Q^{\epsilon}}\left(x^{\mathrm{r},(\ell)}\right)}{f^{P^{\epsilon}}\left(x^{\mathrm{r},(\ell)}\right)}\right)+\frac{1}{\sigma}\right)-\mathrm{Q}\left(\frac{1}{\sigma}\right)\right) \cdot \overline{\mathbb{U}}\left(\ln \left(\frac{f^{Q^{\epsilon}}\left(x^{\mathrm{r},(\ell)}\right)}{f^{P^{\epsilon}}\left(x^{\mathrm{r},(\ell)}\right)}\right)\right)
\end{aligned}
$$

Table I: $\tilde{\mu}_{i, j}^{\mathrm{r},(\ell)}$ for row-type $\mathrm{CN}$ operation (row decoding for PC) over iteration $\ell$, based on the corresponding BDD output $\mu_{i, j}^{r,(\ell)}$ and channel output hard decision $\hat{l}_{i, j}$.

\begin{tabular}{ccc}
\hline $\bar{\mu}_{i, j}^{\mathrm{r},(\ell)}$ & $\hat{l}_{i, j}$ & $\tilde{\mu}_{i, j}^{\mathrm{r},(\ell)}$ \\
\hline-1 & -1 & $\ln \frac{f^{P^{\mathrm{e}}\left(x^{\mathrm{c},(\ell-1)}\right)}}{f^{Q^{\mathrm{c}}\left(x^{\mathrm{c},(\ell-1)}\right)}}$ \\
1 & -1 & $\ln \frac{f^{P^{\mathrm{c}}\left(x^{\mathrm{c},(\ell-1)}\right)}}{f^{Q^{\mathrm{e}}\left(x^{\mathrm{c},(\ell-1)}\right)}}$ \\
0 & -1 & $\ln \frac{f^{P^{\epsilon}}\left(x^{\mathrm{c},(\ell-1)}\right)}{f^{Q^{\epsilon}\left(x^{\mathrm{c},(\ell-1)}\right)}}$ \\
-1 & 1 & $\ln \frac{f^{Q^{\mathrm{e}}}\left(x^{\mathrm{c},(\ell-1)}\right)}{f^{P^{\mathrm{c}}\left(x^{\mathrm{c},(\ell-1)}\right)}}$ \\
1 & 1 & $\ln \frac{f^{Q^{\mathrm{c}}}\left(x^{\mathrm{c},(\ell-1)}\right)}{f^{P^{\mathrm{e}}\left(x^{\mathrm{c},(\ell-1)}\right)}}$ \\
0 & 1 & $\ln \frac{f^{Q^{\epsilon}\left(x^{\mathrm{c},(\ell-1)}\right)}}{f^{P^{\epsilon}}\left(x^{\mathrm{c},(\ell-1)}\right)}$ \\
\hline
\end{tabular}

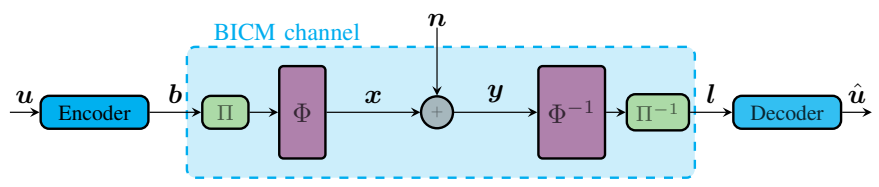

Fig. 3: Summary of the notation for the BICM scheme. The message $\boldsymbol{u}$ is encoded to $\boldsymbol{b}$. After interleaving and mapping, $\boldsymbol{x}$ is sent through a Gaussian channel where the noise $\boldsymbol{n}$ is added, resulting in $\boldsymbol{y}$. The LLRs corresponding to $\boldsymbol{b}$ are denoted as $\boldsymbol{l}$, whereas the decoder output is $\hat{\boldsymbol{u}}$. $\Phi$ maps $m$ bits to a modulation symbol and $\Phi^{-1}$ maps a symbol to $m$ bits.

the resulting $M^{2}$-QAM constellation energy to 1 . In the following, we only consider transmission of the real part of $M^{2}$-QAM, as the real and imaginary parts can be treated independently for the AWGN channel. We consider binary reflected Gray coding (BRGC) mapping [25]. The output of the AWGN channel at time instant $i$ corresponding to ASK symbol $x_{i}$ is given by

$$
y_{i}=x_{i}+n_{i}
$$

Table II: $\tilde{\mu}_{i, j}^{\mathrm{c},(\ell)}$ for column-type CN operation (column decoding for PC) over iteration $\ell$, based on the corresponding BDD output $\mu_{i, j}^{c,(\ell)}$ and channel output hard decision $\hat{l}_{i, j}$.

\begin{tabular}{ccc}
\hline $\bar{\mu}_{i, j}^{\mathrm{c},(\ell)}$ & $\hat{l}_{i, j}$ & $\tilde{\mu}_{i, j}^{\mathrm{c},(\ell)}$ \\
\hline-1 & -1 & $\ln \frac{f^{P^{\mathrm{e}}\left(x^{\mathrm{r},(\ell)}\right)}}{f^{Q^{\mathrm{c}}\left(x^{\mathrm{r},(\ell)}\right)}}$ \\
1 & -1 & $\ln \frac{f^{P^{\mathrm{c}}\left(x^{\mathrm{r},(\ell)}\right)}}{f^{Q^{\mathrm{e}}\left(x^{\mathrm{r},(\ell)}\right)}}$ \\
0 & -1 & $\ln \frac{f^{P^{\epsilon}\left(x^{\mathrm{r},(\ell)}\right)}}{f^{Q^{\epsilon}\left(x^{\mathrm{r},(\ell)}\right)}}$ \\
-1 & 1 & $\ln \frac{f^{Q^{\mathrm{e}}\left(x^{\mathrm{r},(\ell)}\right)}}{f^{P^{\mathrm{c}}\left(x^{\mathrm{r},(\ell)}\right)}}$ \\
1 & 1 & $\ln \frac{f^{Q^{\mathrm{c}}\left(x^{\mathrm{r},(\ell)}\right)}}{f^{P^{\mathrm{e}}\left(x^{\mathrm{r},(\ell)}\right)}}$ \\
0 & 1 & $\ln \frac{f^{Q^{\epsilon}\left(x^{\mathrm{r},(\ell)}\right)}}{f^{P^{\epsilon}\left(x^{\mathrm{r},(\ell)}\right)}}$ \\
\hline
\end{tabular}

where $x_{i} \in \mathcal{X}$ and $n_{i} \sim \mathcal{N}\left(0, \sigma^{2}\right)$. The LLR of the $k$-th bit level of $y_{i}$ is given as

$$
l_{i}^{k}=\ln \left(\frac{\sum_{a \in \mathcal{S}_{k}^{0}} e^{-\frac{\left(y_{i}-a\right)^{2}}{2 \sigma^{2}}}}{\sum_{a \in \mathcal{S}_{k}^{1}} e^{-\frac{\left(y_{i}-a\right)^{2}}{2 \sigma^{2}}}}\right), k=1, \cdots, m
$$

where $\mathcal{S}_{k}^{0} \subset \mathcal{X}$ and $\mathcal{S}_{k}^{1} \subset \mathcal{X}$ are sets of size $2^{m}$ ASK symbols with 0 and 1 as the $k$ th bit of the corresponding BRGC label, respectively. To alleviate the complexity of the LLR computation the well-known max-log approximation is usually used, yielding [26, Eq. 6]

$$
\begin{aligned}
l_{i}^{k} & \approx \frac{1}{2 \sigma^{2}}\left[\max _{a \in \mathcal{S}_{l}^{0}}\left\{-\left(y_{i}-a\right)^{2}\right\}-\max _{a \in \mathcal{S}_{l}^{1}}\left\{-\left(y_{i}-a\right)^{2}\right\}\right] \\
& =\frac{1}{2 \sigma^{2}}\left[\min _{a \in \mathcal{S}_{l}^{1}}\left\{\left(y_{i}-a\right)^{2}\right\}-\min _{a \in \mathcal{S}_{l}^{0}}\left\{\left(y_{i}-a\right)^{2}\right\}\right] .
\end{aligned}
$$




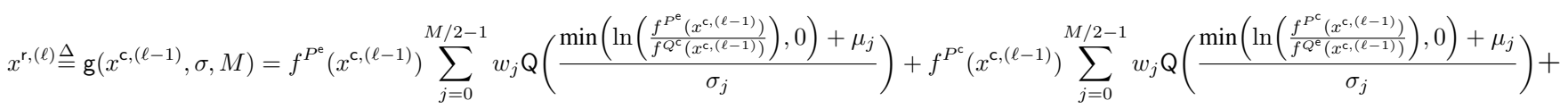

$$
\begin{aligned}
& f^{P^{e}}\left(x^{c,(\ell-1)}\right) \sum_{j=0}^{M / 2-1} w_{j} Q\left(\frac{\min \left(\ln \left(\frac{f^{P^{e}}\left(x^{c,(\ell-1)}\right)}{f^{Q^{e}}\left(x^{,, \ell(-1)}\right)}\right), 0\right)+\mu_{j}}{\sigma_{j}}\right)+f^{Q^{e}}\left(x^{c,(\ell-1)}\right)\left(\sum_{j=0}^{M / 2-1} w_{j}\left(Q\left(\frac{\ln \left(\frac{f^{Q^{e}}\left(x^{c,(\ell-1)}\right)}{f^{P C}\left(x^{c,(\ell-1)}\right)}\right)+\mu_{j}}{\sigma_{j}}\right)-Q\left(\frac{\mu_{j}}{\sigma_{j}}\right)\right)\right) \cdot \overline{\mathbb{U}}\left(\ln \left(\frac{f^{Q^{e}}\left(x^{c,(\ell-1)}\right)}{f^{P^{c}}\left(x^{c,(\ell-1)}\right)}\right)\right) \\
& +f^{Q^{c}}\left(x^{c,(\ell-1)}\right)\left(\sum_{j=0}^{M / 2-1} w_{j}\left(Q\left(\frac{\ln \left(\frac{f^{Q^{C}}\left(x^{c,(\ell-1)}\right)}{f^{P^{e}}\left(x^{c,(\ell-1)}\right)}\right)+\mu_{j}}{\sigma_{j}}\right)-Q\left(\frac{\mu_{j}}{\sigma_{j}}\right)\right)\right) \cdot \overline{\mathbb{U}}\left(\ln \left(\frac{f^{Q^{c}}\left(x^{c,(\ell-1)}\right)}{f^{P^{e}}\left(x^{c,(\ell-1)}\right)}\right)\right) \\
& +f^{Q^{\epsilon}}\left(x^{c,(\ell-1)}\right)\left(\sum _ { j = 0 } ^ { M / 2 - 1 } w _ { j } ( Q ( \frac { \operatorname { l n } ( \frac { f ^ { Q ^ { \epsilon } } ( c ^ { c , ( \ell - 1 ) } ) } { f ^ { P x ^ { c } ( x ^ { c , \ell - 1 ) } ) } ) } ) + \mu _ { j } } { \sigma _ { j } } ) - Q ( \frac { \mu _ { j } } { \sigma _ { j } } ) ) \left(\cdot \overline{\mathbb{U}}\left(\ln \left(\frac{f^{Q^{\epsilon}}\left(x^{c,(\ell-1)}\right)}{f^{P^{\epsilon}}\left(x^{c,(\ell-1)}\right)}\right)\right)\right.\right.
\end{aligned}
$$

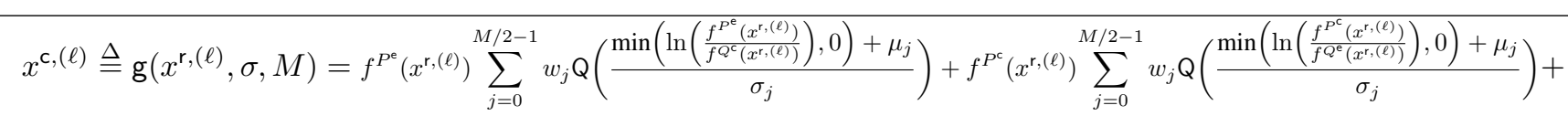

$$
\begin{aligned}
& f^{P^{e}}\left(x^{r,(\ell)}\right) \sum_{j=0}^{M / 2-1} w_{j} \mathrm{Q}\left(\frac{\min \left(\operatorname { l n } \left(\frac{f^{P^{\epsilon}}\left(r^{r,(\ell)}\right)}{\left.\left.f^{Q^{(}\left(r^{r,(\ell)}\right)}\right), 0\right)+\mu_{j}}\right.\right.}{\sigma_{j}}\right)+f^{Q^{e}}\left(x^{r,(\ell)}\right)\left(\sum_{j=0}^{M / 2-1} w_{j}\left(Q\left(\frac{\ln \left(\frac{f^{Q^{e}}\left(x^{r,(\ell)}\right)}{f^{P^{c}}\left(x^{r,(\ell)}\right)}\right)+\mu_{j}}{\sigma_{j}}\right)-Q\left(\frac{\mu_{j}}{\sigma_{j}}\right)\right)\right) \cdot \overline{\mathbb{U}}\left(\ln \left(\frac{f^{Q^{e}}\left(x^{r,(\ell)}\right)}{f^{P^{c}}\left(x^{r,(\ell)}\right)}\right)\right) \\
& +f^{Q^{c}}\left(x^{r,(\ell)}\right)\left(\sum_{j=0}^{M / 2-1} w_{j}\left(Q\left(\frac{\ln \left(\frac{f^{Q^{c}}\left(x^{r,(\ell)}\right)}{f^{P^{(}}\left(x^{r,(\ell)}\right)}\right)+\mu_{j}}{\sigma_{j}}\right)-Q\left(\frac{\mu_{j}}{\sigma_{j}}\right)\right)\right) \cdot \overline{\mathbb{U}}\left(\ln \left(\frac{f^{Q^{c}}\left(x^{r,(\ell)}\right)}{f^{P^{e}}\left(x^{r,(\ell)}\right)}\right)\right) \\
& +f^{Q^{\epsilon}}\left(x^{r,(\ell)}\right)\left(\sum_{j=0}^{M / 2-1} w_{j}\left(Q\left(\frac{\ln \left(\frac{f^{Q^{\epsilon}}\left(x^{r,(\ell)}\right)}{f^{P^{\epsilon}(}\left(x^{r,(\ell \ell)}\right)}\right)+\mu_{j}}{\sigma_{j}}\right)-Q\left(\frac{\mu_{j}}{\sigma_{j}}\right)\right)\right) \cdot \overline{\mathbb{U}}\left(\ln \left(\frac{f^{Q^{\epsilon}}\left(x^{r,(\ell)}\right)}{f^{P^{\epsilon}}\left(x^{r,(\ell)}\right)}\right)\right)
\end{aligned}
$$

$$
p(l \mid b)=\sum_{j=0}^{\frac{M}{2}-1} w_{j} \mathrm{G}\left(l ;(-1)^{b} \mu_{j}, \sigma_{j}^{2}\right),
$$

where $l$ is the LLR corresponding to transmitted bit $b \in\{0,1\}$, $w_{j}=\frac{2\left(2^{m-\left\lceil\log _{2}(j+1)\right\rceil}-1\right)}{m \cdot M}, \mu_{j}=\frac{2 \Delta^{2}(j+1)^{2}}{\sigma^{2}}$, and $\sigma_{j}^{2}=$ $\frac{4 \Delta^{2}(j+1)^{2}}{\sigma^{2}}$, respectively. To proceed with the DE analysis, we assume that the all-zero codeword is transmitted. This leads to the need to symmetrizing the LLR distribution. To achieve this, we resort to the use of channel adapters [27]. Denote by $\bar{l}$ the LLR of the symmetrized BICM channel. The distribution of $\bar{l}$ is given as [28, Eq. (19)]

$$
p(\bar{l} \mid b)=\frac{p(l \mid b)+p(-l \mid 1-b)}{2} .
$$

Proposition 2: For a BICM scheme with BRGC mapping, the message error probability at the row-type and column-type $\mathrm{CN}$ at the $\ell$ th iteration is given by (13) and (14), respectively, with

$$
x^{c,(0)}=\sum_{j=0}^{\frac{M}{2}-1} w_{j} \cdot \mathrm{Q}\left(\frac{\mu_{j}}{\sigma_{j}}\right) .
$$

The proof is given in Appendix B

\section{Numerical RESUlts}

In this section, in order to have a direct comparison with iBDD-SR, we consider PCs with the same component codes as those considered in $[8]$, i.e., PCs with $(255,231,3)$ and $(511,484,3) \mathrm{BCH}$ component codes 5 The code rates of the

\footnotetext{
${ }^{5}$ Component codes with long block length and $t=3$ are interesting for fiber-optic communications as their error floor is below $10^{-15}$ and the decoder can be efficiently implemented using LUTs [5. Appendix I].
}

PCs are $R=0.820$ and 0.897 , respectively. Also, we consider 12 decoding iterations.

Remark 4: In the case of errors with high reliability, i.e., LLRs with high magnitude and wrong sign, the decoding rule in (3)-(4) will be unable to correct such errors, as it is likely that $\mathrm{B}\left(\tilde{l}_{i, j}^{\mathfrak{r},(\ell)}\right)=\mathrm{B}\left(l_{i, j}\right)$. Therefore, similar to iBDDSR [8, Sec. VI], one should selectively apply iBDD-CR to to increase the chance of correcting such errors. In particular, unless otherwise specified, we consider iBDD-CR and iBDDSR for some iterations and then we append a few conventional iBDD iterations. The additional iBDD iterations increase the chance of correcting transmission errors with high reliability, as the iBDD decoding rule is independent of channel reliabilities. Specifically, we consider a maximum of 10 iBDDCR (or iBDD-SR) iterations followed by 2 conventional iBDD iterations. For the sake of fairness, other decoding algorithms are evaluated with 12 decoding iterations.

We highlight that for the PC performance simulation, we obtain the values of $\tilde{\mu}_{i, j}^{\mathrm{r},(\ell)}$ and $\tilde{\mu}_{i, j}^{\mathrm{c},(\ell)}$ from the DE for a single $E_{\mathrm{b}} / N_{0}$, corresponding to an SNR point in the waterfall region. We found that changing the operating SNR point in the waterfall region (which in principle results in different values for $\tilde{\mu}_{i, j}^{r,(\ell)}$ and $\left.\tilde{\mu}_{i, j}^{\mathrm{c},(\ell)}\right)$ yields a minor performance difference in the simulation results. Furthermore, in the following simulation results we restrict to $\tilde{\mu}_{i, j}^{\mathrm{r},(\ell)}=\tilde{\mu}_{i, j}^{\mathrm{c},(\ell)}$, i.e., for a given decoding iteration the values of $\tilde{\mu}_{i, j}^{r,(\ell)}$ in Table $\mathrm{I}$ are used for both row and column decoding, as we found that this induces a negligible performance loss.

In Fig. 4, the performance of PCs with iBDD-CR and iBDD-SR over the bi-AWGN channel is shown and compared with the DE thresholds of the corresponding GLDPC code ensembles. As it can be seen, the performance improvement of iBDD-CR over iBDD-SR is well-predicted by the DE 


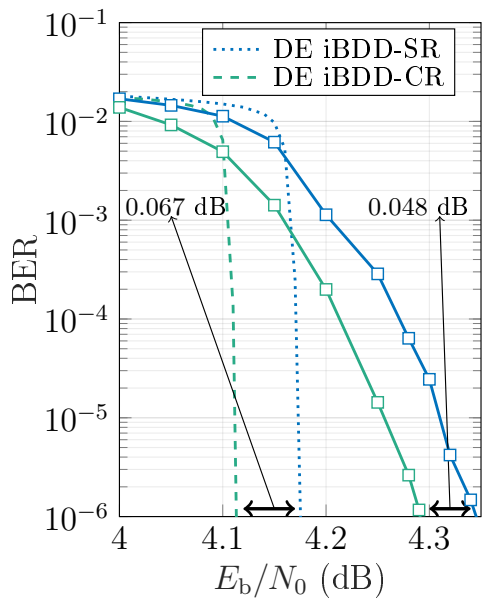

(a)

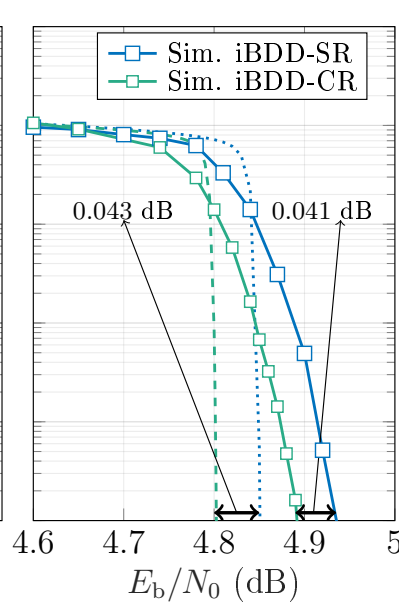

(b)
Fig. 4: Comparison between DE thresholds (computed for the relevant GLDPC ensembles) and BER performance of iBDD-CR and iBDD-SR algorithms applied to PCs. The BCH component codes for (a) and (b) have parameters $(255,231,3)$ and $(511,484,3)$, respectively.

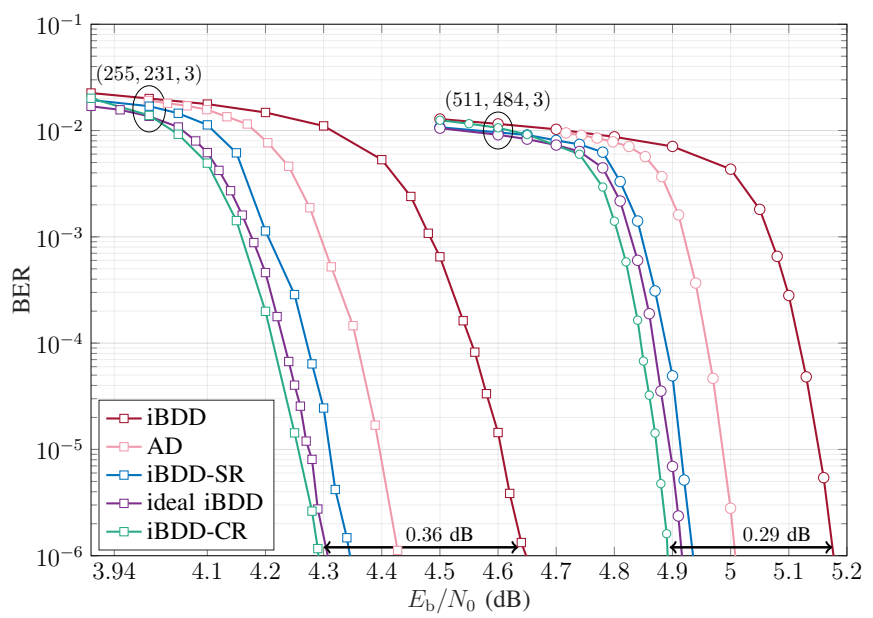

Fig. 5: Performance of iBDD, ideal iBDD, AD, iBDD-SR, and iBDD-CR for a PC with a $(255,231,3) \mathrm{BCH}$ component code and a staircase code with a $(511,484,3) \mathrm{BCH}$ component code.

analysis, confirming that DE can be used for as a tool for the optimization of the decoding algorithm. The gap between the DE thresholds and the BER curves is due to two reasons. First and foremost, DE predicts the performance of the GLDPC code ensemble with infinite long block length. By increasing the PC component code block length from 255 to 511, the gap is reduced, as it can be seen in Fig. 4. Second, DE analysis relies on an extrinsic message passing variation of the algorithms, whereas the simulation results employ standard (intrinsic) message passing.

In Fig. 5, we show the performance of iBDD-CR, iBDD, ideal iBDD, anchor decoding (AD) [7], and iBDD-SR for transmission over the bi-AWGN channel. One can see that iBDD-CR outperforms all other decoders. In particular, iBDDCR performs even better than ideal iBDD. The performance gain of iBDD-CR over iBDD is $0.36 \mathrm{~dB}$ and $0.29 \mathrm{~dB}$ for PCs with component codes $(255,231,3)$ and $(511,484,3)$, respectively. In Table III we show the required $E_{\mathrm{b}} / N_{0}$ for iBDD$\mathrm{CR}$ and iBDD to achieve a BER of $10^{-6}$. We also show
Table III: Comparison of iBDD-CR and iBDD for PCs with $(255,231,3)$ and $(511,484,3) \mathrm{BCH}$ component codes, with code rates of 0.820 and 0.897 , respectively. The $E_{\mathrm{b}} / N_{0}$ for $\mathrm{BBDD}$ and $\mathrm{iBDD}-\mathrm{CR}$ are measured at BER $=$ $10^{-6}$ from the simulations. The corresponding Shannon limits are also shown.

\begin{tabular}{cccc}
\hline $\begin{array}{c}\text { component } \\
\text { code }\end{array}$ & $\begin{array}{c}\text { decoding } \\
\text { algorithm }\end{array}$ & $E_{\mathrm{b}} / N_{0}[\mathrm{~dB}]$ & Shannon limit [dB] \\
\hline$(255,231,3)$ & iBDD & 4.62 & $3.54(\mathrm{HD})$ \\
$(255,231,3)$ & iBDD-CR & 4.29 & $2.23(\mathrm{SD})$ \\
$(511,484,3)$ & iBDD & 5.18 & $4.36(\mathrm{HD})$ \\
$(511,484,3)$ & iBDD-CR & 4.89 & $3.15(\mathrm{SD})$ \\
\hline
\end{tabular}

the corresponding hard-decision (HD) and soft-decision (SD) Shannon limits. Note that iBDD should be compared with the HD Shannon limit, while iBDD-CR should be compared with the SD counterpart, as the algorithm exploits the channel LLRs. The gap between the performance of iBDD-CR and the SD Shannon limit is mainly due to the fact that the structure of iBDD-CR structure is intentionally kept very similar to that of iBDD, in order to constrain the decoder complexity and the internal decoder data flow. By allowing the exchange of soft information between component decoders this gap can be closed further at the cost of significantly higher data flow and complexity (see [9]-[11] for more details).

In Fig. 6, we show the performance of a BICM system using a PC with component code $(255,231,3)$ for iBDD, ideal iBDD, iBDD-SR, and iBDD-CR and 16-QAM, 64-QAM, and 256QAM. We also show the DE threshold for the corresponding GLDPC ensemble. iBDD-CR outperforms others decoders and the DE analysis predicts the performance of iBDD-CR accurately. Furthermore, the gain of iBDD-CR over iBDD improves by increasing the modulation order; the gain is up to $0.51 \mathrm{~dB}$ for 256 -QAM.

In Fig. 7) we examine the effect of appended iBDD iterations on the performance of iBDD-CR. We split the total of 12 decoding iterations between iBDD-CR and iBDD. As it can be seen, increasing the number of iBDD-CR iterations yields a performance improvement, where such improvement saturates at 10 iBDD-CR and 2 iBDD iterations. This motivates the choice of appending 2 iBDD iterations for performance evaluation of iBDD-CR (see Remark 4).

To evaluate the sensitivity of iBDD-CR to channel LLR quantization, we resort to a classical quantization scheme called Lloyd-Max algorithm [29], [30], which aims at optimizing the quantization levels in the sense of minimizing the mean squared error between LLRs and the corresponding quantized values ${ }^{6}$ As an example, Fig. 8 shows the distribution of LLRs for 16-QAM at $E_{\mathrm{s}} / N_{0}=12.92 \mathrm{~dB} 7$ the optimized quantization values, and the corresponding boundaries using 3 bits based on the Lloyd-Max algorithm. As it can be seen, Lloyd-Max yields nonuniform quantization values.

In Fig. 9, we investigate the effect of max-log channel LLR approximation and channel LLR quantization based on the Lloyd-Max algorithm on the performance of iBDD-CR.

\footnotetext{
${ }^{6} \mathrm{We}$ highlight that in this paper our approach is to just show the feasibility of iBDD-CR implementation with limited channel LLR quantization levels, using a known quantization technique. In general, one can exploit the properties of the quantized channel in decoding rule to reduce the sensitivity to quantization errors. This analysis is beyond the scope of this paper.

${ }^{7}$ We highlight that $E_{\mathrm{s}} / N_{0}=12.92 \mathrm{~dB}$ corresponds to $E_{\mathrm{b}} / N_{0}=7.76 \mathrm{~dB}$ which is a point selected in the waterfall region of iBDD-CR (see Fig. 7 .
} 


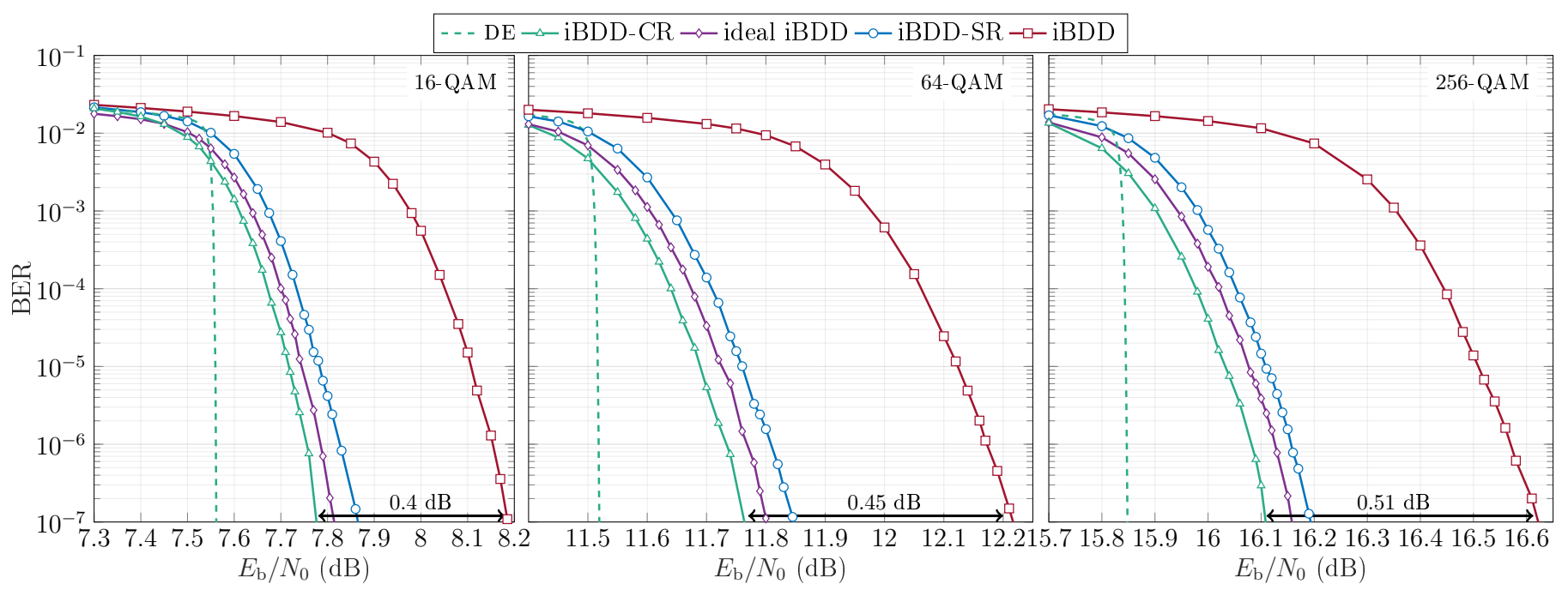

Fig. 6: Performance of iBDD, ideal iBDD, iBDD-SR, and iBDD-CR with unquantized LLRs for a $(255,231,3)$ BCH component code in a BICM scheme with 16-QAM, 64-QAM, and 256-QAM modulation.

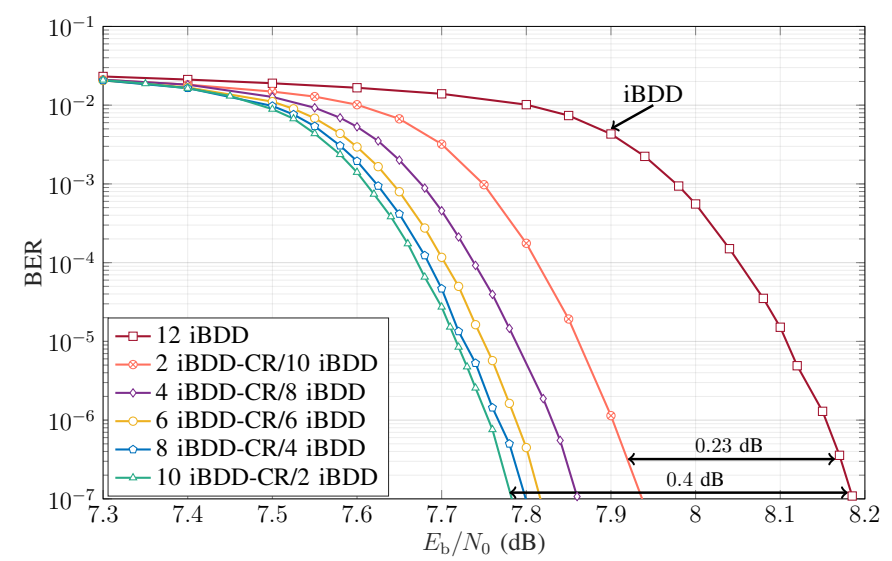

Fig. 7: Performance of iBDD-CR with different appended iBDD iterations for the BICM channel with 16-QAM modulation and $\mathrm{PC}$ with $\mathrm{BCH}$ component code $(255,231,3)$

In particular, we consider a BICM channel with 16-QAM modulation, PC with BCH component code $(255,231,3)$, and 3-bit and 4-bit quantization. One can see that the performance of iBDD-CR with exact channel LLR computation (16) and max-log channel LLR approximation (17) is almost identical, hence, in a practical system max-log approximation can be employed to reduce the complexity of LLR computations. Furthermore, at a BER of $10^{-7}$, the performance loss of iBDDCR with 3 and 4 bits nonuniform channel LLR quantization based on the Lloyd-Max algorithm is small, i.e., $0.07 \mathrm{~dB}$ and $0.045 \mathrm{~dB}$, respectively. This shows that iBDD-CR has a low sensitivity to channel LLR quantization.

\section{CONCLUSION}

We proposed an iterative soft-aided decoding algorithm for PCs, called iBDD-CR. iBDD-CR exploits the LLRs in the BDD of the component codes and has the same decoder data flow as that of conventional iBDD. We performed a DE analysis of the GLDPC code ensemble containing PCs for both the bi-AWGN and BICM channels under extrinsic mes-

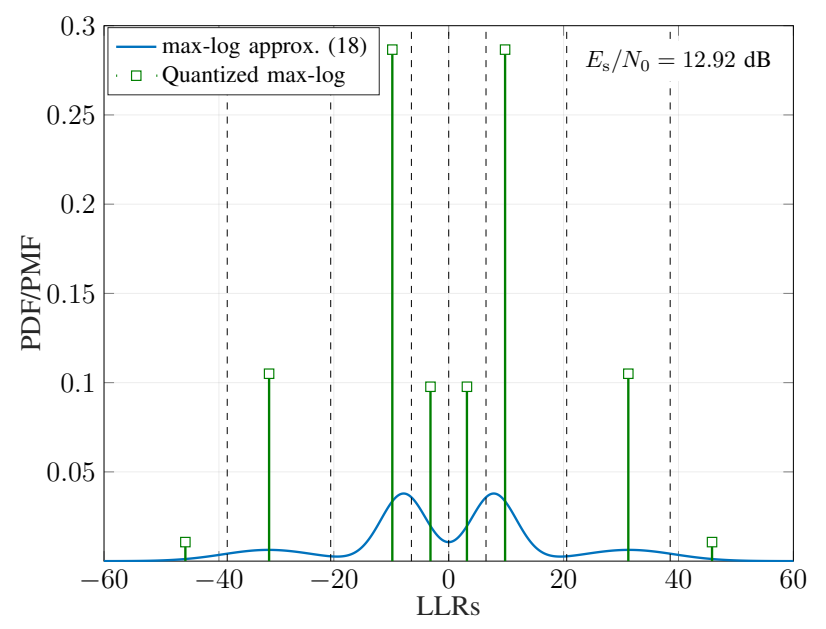

Fig. 8: Comparison between the PDF of the LLRs and the corresponding quantized PMF using 3 bits for 16-QAM modulation and $E_{\mathrm{s}} / N_{0}=12.92$ $\mathrm{dB}$. The dashed line shows the boundaries of the optimized nonuniform quantization based on the Lloyd-Max algorithm.

sage passing. From the analysis, an accurate estimate of the reliability of the BDD outbound messages, which is essential for implementing the iBDD-CR was derived. The proposed algorithm attains gains up to $0.51 \mathrm{~dB}$ over conventional iBDD and outperforms ideal iBDD. We showed that iBDD-CR has a low sensitivity to quantization errors on the channel LLRs and can be implemented using the low-complexity max-log LLR approximation. Overall, the low required internal data flow and low sensitivity to quantization errors of iBDD-CR makes it an attractive solution for optical communication for 400G and beyond, where an excellent performance along with stringent constraint on latency and power consumption are required.

We remark that iBDD-CR requires some extra memory compared to iBDD to store the channel LLRs and the LUTs. The exact required extra memory depends on the level of parallelism in implementing row/column decoding and the relative required clock cycles of BDD and combining stages. Therefore, the complexity of iBDD-CR should be investigated via hardware implementation, which is left as future work. 


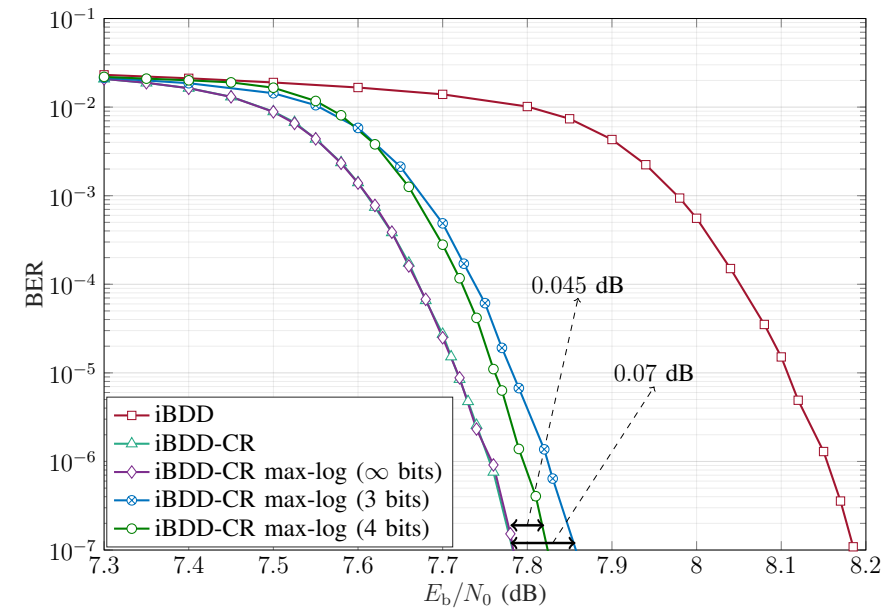

Fig. 9: Performance of iBDD-CR with exact, max-log approximation, and quantized channel LLRs for the BICM channel with 16-QAM modulation and $\mathrm{PC}$ with $\mathrm{BCH}$ component code $(255,231,3)$.

\section{ACKNOWLEDGMENT}

The authors would like to thank Dr. Alexios BalatsoukasStimming for fruitful discussions about the complexity of iBDD-CR.

\section{APPENDIX A}

\section{PROOF OF PROPOSITION 1}

Let us consider the decoding of row-type CNs. In particular, we first compute $\tilde{\mu}_{i, j}^{r,(\ell)}$ and then we calculate $x^{r,(\ell)}$. At the first iteration, we have $x^{\mathrm{c},(0)}=p_{\mathrm{ch}}$, i.e., the input of the row-type $\mathrm{CN}$ is initialized with the channel error probability. Let $\hat{l}_{i, j}$ be the RV representing the sign of the LLR corresponding to code bit $c_{i, j}$ (see Fig. 1). To compute $\tilde{\mu}_{i, j}^{r,(\ell)}$ (see (8)), we should compute the probabilities of $p\left(\bar{\mu}_{i, j}^{\mathrm{r},(\ell)} \mid \hat{l}_{i, j}, c_{i, j}=0\right)$ and $p\left(\bar{\mu}_{i, j}^{\mathrm{r},(\ell)} \mid \hat{l}_{i, j}, c_{i, j}=1\right)$. Depending on the values of $\hat{l}_{i, j} \in\{ \pm 1\}$ and $\mu_{i, j}^{r,(\ell)} \in\{0, \pm 1\}$, six different terms for $p\left(\bar{\mu}_{i, j}^{\mathrm{r},(\ell)} \mid \hat{l}_{i, j}, c_{i, j}=0\right)$ and $p\left(\bar{\mu}_{i, j}^{\mathrm{r},(\ell)} \mid \hat{l}_{i, j}, c_{i, j}=1\right)$ are possible (12 in total). The term $p\left(\bar{\mu}_{i, j}^{r,(\ell)}=-1 \mid \hat{l}_{i, j}=-1, c_{i, j}=0\right)$ is the probability of error at the BDD output given that the channel output is also in error. One can check that this is exactly the definition of $p\left(\bar{\mu}_{i, j}^{\mathrm{r},(\ell)}=1 \mid \hat{l}_{i, j}=1, c_{i, j}=1\right)$ as the values for $\bar{\mu}_{i, j}^{\mathrm{r},(\ell)}$ and $\hat{l}_{i, j}$ are changed from -1 to 1 and the value for $c_{i, j}$ is changed from 0 to 1 . Therefore, $p\left(\bar{\mu}_{i, j}^{\mathrm{r},(\ell)}=-1 \mid \hat{l}_{i, j}=-1, c_{i, j}=0\right)=p\left(\bar{\mu}_{i, j}^{\mathrm{r},(\ell)}=1 \mid \hat{l}_{i, j}=1, c_{i, j}=1\right)$. Similarly, the following relations also hold:

$p\left(\bar{\mu}_{i, j}^{\mathrm{r},(\ell)}=1 \mid \hat{l}_{i, j}=1, c_{i, j}=0\right)=p\left(\bar{\mu}_{i, j}^{\mathrm{r},(\ell)}=-1 \mid \hat{l}_{i, j}=-1, c_{i, j}=1\right)$, $p\left(\bar{\mu}_{i, j}^{\mathrm{r},(\ell)}=1 \mid \hat{l}_{i, j}=-1, c_{i, j}=0\right)=p\left(\bar{\mu}_{i, j}^{r,(\ell)}=-1 \mid \hat{l}_{i, j}=1, c_{i, j}=1\right)$, $p\left(\bar{\mu}_{i, j}^{\mathrm{r},(\ell)}=-1 \mid \hat{l}_{i, j}=1, c_{i, j}=0\right)=p\left(\bar{\mu}_{i, j}^{r,(\ell)}=1 \mid \hat{l}_{i, j}=-1, c_{i, j}=1\right)$, $p\left(\bar{\mu}_{i, j}^{r,(\ell)}=0 \mid \hat{l}_{i, j}=-1, c_{i, j}=0\right)=p\left(\bar{\mu}_{i, j}^{r,(\ell)}=0 \mid \hat{l}_{i, j}=1, c_{i, j}=1\right)$, and $p\left(\bar{\mu}_{i, j}^{\mathrm{r},(\ell)}=0 \mid \hat{l}_{i, j}=1, c_{i, j}=0\right)=p\left(\bar{\mu}_{i, j}^{\mathrm{r},(\hat{\ell})}=0 \mid \hat{l}_{i, j}=-1, c_{i, j}=1\right)$.

We assume all-zero codeword transmission. Such assumption yields the computation of six different terms corresponding to $p\left(\bar{\mu}_{i, j}^{\mathrm{r},(\ell)} \mid \hat{l}_{i, j}, c_{i, j}=0\right)$.

Let $x$ be the input error probability to the row-type CNs for the BDD stage and denote by $f^{P^{\mathrm{e}}}(x)$ the probability that a randomly selected bit in the component code's codeword is decoded incorrectly when it was initially in error. The notion of randomly selected bit is motivated due to the fact that we analyze the ensemble given in Fig. 2. (c), where the connection between $\mathrm{VN}$ and $\mathrm{CN}$ is randomly built. Also, we use the notion of initially in error as we analyze the extrinsic message passing where in each iteration the input corresponding to $c_{i, j}$ is substituted by channel input $r_{i, j}$ (see (5)). Furthermore, we denote by $P^{\mathrm{e}}(i)$ the probability that a randomly selected bit in the component code's codeword is decoded incorrectly when it was initially in error and there are $i$ errors in the other $n-1$ positions. $P^{\mathrm{e}}(i)$ was derived in [8, Eq. (5)] for iBDD$\mathrm{SR}$ and the same expression holds for $\mathrm{BBDD}-\mathrm{CR} . f^{P^{\mathrm{e}}}(x)$ is then obtained based on the union of (independent) events for $i \in\{0, \cdots, n-1\}$ random errors as

$$
f^{P^{\mathrm{e}}}(x) \triangleq p\left(\bar{\mu}_{i, j}^{\mathrm{r},(\ell)}=-1 \mid \hat{l}_{i, j}=-1, c_{i, j}=0\right)=\sum_{i=0}^{n-1} b_{i}^{n}(x) P^{\mathrm{e}}(i) .
$$

where $b_{i}^{n}(x) \triangleq\left(\begin{array}{c}n-1 \\ i\end{array}\right) x^{i}(1-x)^{n-i-1}$.

We denote by $f^{P^{c}}(x)$ and $f^{P^{\epsilon}}(x)$ the probability that a randomly selected bit in the component code's codeword is decoded correctly and erased ${ }^{8}$ respectively, when it was initially in error. Furthermore, we denote by $P^{\mathrm{c}}(i)$ and $P^{\epsilon}(i)$ the probability that a randomly selected bit in the component code's codeword is decoded correctly and erased, respectively, when it was initially in error and there are $i$ errors in the other $n-1$ positions. $P^{\mathrm{c}}(i)$ is given in [8. Eq. (9)] and $P^{\epsilon}(i)=1-P^{\mathrm{e}}(i)-P^{\mathrm{c}}(i)$. Similar to the derivation of $(20)$, $f^{P^{c}}(x)$ and $f^{P^{\epsilon}}(x)$ are obtained as

$$
\begin{aligned}
& f^{P^{\mathrm{c}}}(x) \triangleq p\left(\bar{\mu}_{i, j}^{\mathrm{r},(\ell)}=1 \mid \hat{l}_{i, j}=-1, c_{i, j}=0\right)=\sum_{i=0}^{n-1} b_{i}^{n}(x) P^{\mathrm{c}}(i), \\
& f^{P^{\epsilon}}(x) \triangleq p\left(\bar{\mu}_{i, j}^{\mathrm{r},(\ell)}=0 \mid \hat{l}_{i, j}=-1, c_{i, j}=0\right)=\sum_{i=0}^{n-1} b_{i}^{n}(x) P^{\epsilon}(i) .
\end{aligned}
$$

Furthermore, we denote by $f^{Q^{\mathrm{e}}}(x), f^{Q^{\mathrm{c}}}(x)$, and $f^{Q^{\epsilon}}(x)$ the probability that a randomly selected bit in the component code's codeword is decoded incorrectly, correctly, and erased, respectively, when the bit was initially correct. Also, let us denote by $Q^{\mathrm{e}}(i), Q^{\mathrm{c}}(i)$, and $Q^{\epsilon}(i)$ the probability that a randomly selected bit in the component code's codeword is decoded incorrectly, correctly, and erased, respectively, when the bit was initially correct and there are $i$ errors in the remaining $n-1$ positions. $Q^{\mathrm{c}}(i)$ is given in [8, Eq. (6)], $Q^{\mathrm{e}}(i)$ in [8. Eq. (10)], and $Q^{\epsilon}(i)=1-Q^{\mathrm{e}}(i)-Q^{\mathrm{c}}(i)$. Following the same steps as for the derivation of 20 , we get

$$
\begin{gathered}
f^{Q^{\mathrm{e}}}(x) \triangleq p\left(\bar{\mu}_{i, j}^{\mathrm{r},(\ell)}=-1 \mid \hat{l}_{i, j}=1, c_{i, j}=0\right)=\sum_{i=0}^{n-1} b_{i}^{n}(x) Q^{\mathrm{e}}(i), \\
f^{Q^{\mathrm{c}}}(x) \triangleq p\left(\bar{\mu}_{i, j}^{\mathrm{r},(\ell)}=1 \mid \hat{l}_{i, j}=1, c_{i, j}=0\right)=\sum_{i=0}^{n-1} b_{i}^{n}(x) Q^{\mathrm{c}}(i), \\
f^{Q^{\epsilon}}(x) \triangleq p\left(\bar{\mu}_{i, j}^{\mathrm{r},(\ell)}=0 \mid \hat{l}_{i, j}=1, c_{i, j}=0\right)=\sum_{i=0}^{n-1} b_{i}^{n}(x) Q^{\epsilon}(i) .
\end{gathered}
$$

${ }^{8}$ Note the $\bar{\mu}_{i, j}^{\mathrm{r},(\ell)}=0$ corresponds to the erasure output of BDD. 
Recalling (8) and the discussion given in the begining of this appendix, by employing 20)-25 and $x^{c,(\ell-1)}$ as the input error probability of row-type $\mathrm{CN}$ at iteration $\ell$, the values of $\tilde{\mu}_{i, j}^{r,(\ell)}$ given in Table $\mathrm{I}$ are obtained.

Now we focus on computing $x^{\mathrm{r},(\ell)}$. Assuming transmission of the all-zero codeword, using (7), and applying Bayes' rule, the probability of error at the output of row decoder is obtained as

$$
\begin{aligned}
x^{\mathrm{r},(\ell)} & =p\left(\widetilde{l}_{i, j}^{\mathrm{r},(\ell)}<0\right) \\
& =\sum_{\substack{\bar{\mu}_{i, j}^{\mathrm{r},(\ell)} \in\{0, \pm 1\} \\
\hat{L}_{i, j} \in\{ \pm 1\}}} p\left(\widetilde{l}_{i, j}^{\mathrm{r},(\ell)}<0 \mid \bar{\mu}_{i, j}^{\mathrm{r},(\ell)}, \hat{l}_{i, j}\right) p\left(\bar{\mu}_{i, j}^{\mathrm{r},(\ell)} \mid \hat{l}_{i, j}\right) p\left(\hat{l}_{i, j}\right)
\end{aligned}
$$

where $p\left(\bar{\mu}_{i, j}^{\mathrm{r},(\ell)} \mid \hat{l}_{i, j}\right)$ is given in 20)-25]. In the following, we compute $p\left(\tilde{l}_{i, j}^{r,(\ell)}<0 \mid \bar{\mu}_{i, j}^{\mathrm{r},(\ell)}, \hat{l}_{i, j}\right)$. In particular, let us first calculate the term $p\left(\tilde{l}_{i, j}^{\mathrm{r},(\ell)}<0 \mid \bar{\mu}_{i, j}^{\mathrm{r},(\ell)}=-1, \hat{l}_{i, j}=-1\right)$ in 26 . This probability can be written as

$$
\begin{aligned}
& p\left(\tilde{l}_{i, j}^{\mathrm{r},(\ell)}<0 \mid \bar{\mu}_{i, j}^{\mathrm{r},(\ell)}=-1, \hat{l}_{i, j}=-1\right) \stackrel{(a)}{=} \\
& p\left(l_{i, j}<-\ln \left(\frac{f^{P^{\mathrm{e}}}\left(x^{\mathrm{c},(\ell-1)}\right)}{\left.f^{Q^{\mathrm{c}}\left(x^{\mathrm{c},(\ell-1)}\right)}\right)}\right) \bar{\mu}_{i, j}^{\mathrm{r},(\ell)}=-1, \hat{l}_{i, j}=-1\right) \stackrel{(b)}{=} \\
& p\left(l_{i, j}<-\ln \left(\frac{f^{P^{\mathrm{e}}}\left(x^{c,(\ell-1)}\right)}{f^{Q^{c}}\left(x^{c,(\ell-1)}\right)}\right) \mid \hat{l}_{i, j}=-1\right) \stackrel{(c)}{=} \\
& \frac{p\left(l_{i, j}<-\ln \left(\frac{f^{P^{\mathrm{e}}}\left(x^{\mathrm{c},(\ell-1)}\right)}{f^{Q^{c}}\left(x^{c,(\ell-1)}\right)}\right), l_{i, j}<0\right)}{p\left(l_{i, j}<0\right)} \stackrel{(d)}{=} \\
& \frac{p\left(l_{i, j}<\min \left(-\ln \left(\frac{f^{P^{\mathrm{e}}}\left(x^{c,(\ell-1)}\right)}{f^{Q^{C}}\left(x^{c,(\ell-1)}\right)}\right), 0\right)\right)}{p\left(l_{i, j}<0\right)} \stackrel{(e)}{=} \\
& \frac{\mathrm{Q}\left(\frac{\sigma}{2} \min \left(\ln \left(\frac{f^{P^{\mathrm{e}}}\left(x^{\mathrm{c},(\ell-1)}\right)}{f^{Q^{c}}\left(x^{c,(\ell-1)}\right)}\right), 0\right)+\frac{1}{\sigma}\right)}{p\left(l_{i, j}<0\right)}
\end{aligned}
$$

where $(a)$ follows from (7) and Table $\mathrm{I}$. $(b)$ follows from the Markov chain $l_{i, j} \rightarrow \hat{l}_{i, j} \rightarrow \bar{\mu}_{i, j}^{\mathrm{r},(\ell)}$ (see Sec. IV-A), (c) follows from the definition of conditional probability and the fact that $p\left(\hat{l}_{i, j}=-1\right)=p\left(l_{i, j}<0\right),(d)$ follows by intersecting the events $\left\{l_{i, j}<-\ln \left(\frac{f^{P^{e}}\left(x^{c,(\ell-1)}\right)}{f^{Q^{c}}\left(x^{c,(\ell-1)}\right)}\right)\right\}$ and $\left\{l_{i, j}<0\right\}$, and $(e)$ follows by recalling that $l_{i, j} \sim \mathcal{N}\left(2 / \sigma^{2}, 4 / \sigma^{2}\right)$ if $c_{i, j}=0$ and employing the $\mathrm{Q}(\cdot)$ function.

With the same approach above, one can compute the following probabilities

$$
\begin{aligned}
& p\left(\tilde{l}_{i, j}^{r,(\ell)}<0 \mid \bar{\mu}_{i, j}^{\mathbf{r},(\ell)}=1, \hat{l}_{i, j}=-1\right)= \\
& \frac{\mathrm{Q}\left(\frac{\sigma}{2} \min \left(\ln \left(\frac{f^{P^{c}}\left(x^{c,(\ell-1)}\right)}{f^{Q}\left(x^{c,(\ell-1)}\right)}\right), 0\right)+\frac{1}{\sigma}\right)}{p\left(l_{i, j}<0\right)}, \\
& p\left(\tilde{l}_{i, j}^{r,(\ell)}<0||_{i, j}^{r,(\ell)}=0, \hat{l}_{i, j}=-1\right)= \\
& \frac{\mathrm{Q}\left(\frac{\sigma}{2} \min \left(\ln \left(\frac{f^{P^{\epsilon}}\left(x^{c,(\ell-1)}\right)}{f^{Q^{\epsilon}}\left(x^{c,(\ell-1)}\right)}\right), 0\right)+\frac{1}{\sigma}\right)}{p\left(l_{i, j}<0\right)} .
\end{aligned}
$$

The probability $p\left(\tilde{l}_{i, j}^{\mathrm{r}(\ell)}<0 \mid \bar{\mu}_{i, j}^{\mathrm{r},(\ell)}=-1, \hat{l}_{i, j}=1\right)$ is

$$
\begin{aligned}
& p\left(\tilde{l}_{i, j}^{\mathrm{r},(\ell)}<0 \mid \bar{\mu}_{i, j}^{\mathrm{r},(\ell)}=-1, \hat{l}_{i, j}=1\right) \stackrel{(a)}{=} \\
& p\left(l_{i, j}<-\ln \left(\frac{f^{Q^{\mathrm{e}}}\left(x^{\mathrm{c},(\ell-1)}\right)}{f^{P^{\mathrm{c}}}\left(x^{\mathrm{c},(\ell-1)}\right)}\right) \mid \bar{\mu}_{i, j}^{\mathrm{r},(\ell)}=-1, \hat{l}_{i, j}=1\right) \stackrel{(b)}{=} \\
& p\left(l_{i, j}<-\ln \left(\frac{f^{Q^{\mathrm{e}}}\left(x^{\mathrm{c},(\ell-1)}\right)}{\left.\left.f^{P^{\mathrm{c}}\left(x^{\mathrm{c},(\ell-1)}\right)}\right) \mid \hat{l}_{i, j}=1\right) \stackrel{(c)}{=}}\right.\right. \\
& \frac{p\left(l_{i, j}<-\ln \left(\frac{f^{Q^{\mathrm{e}}}\left(x^{c,(\ell-1)}\right)}{f^{P^{\mathrm{c}}}\left(x^{c,(\ell-1)}\right)}\right), l_{i, j}>0\right)}{p\left(l_{i, j}>0\right)} \stackrel{(d)}{=} \\
& \frac{p\left(0<l_{i, j}<-\ln \left(\frac{f^{Q^{\mathrm{e}}}\left(x^{c,(\ell-1)}\right)}{f^{P^{\mathrm{c}}}\left(x^{c,(\ell-1)}\right)}\right)\right)}{p\left(l_{i, j}>0\right)}
\end{aligned}
$$

where $(a)$ follows from (7) and Table I] $(b)$ follows from the Markov chain $l_{i, j} \rightarrow \hat{l}_{i, j} \rightarrow \bar{\mu}_{i, j}^{r},(\ell),(c)$ follows from the definition of conditional probability and the fact that $p\left(\hat{l}_{i, j}=1\right)=p\left(l_{i, j}>0\right)$, and $(d)$ follows by intersecting the events $\left\{l_{i, j}<-\ln \left(\frac{f^{Q^{e}}\left(x^{c,(\ell-1)}\right)}{f^{P^{C}}\left(x^{c, \ell-1)}\right)}\right)\right\}$ and $\left\{l_{i, j}>0\right\}$. Note that we assumed that $\ln \left(\frac{f^{Q^{\ell}}\left(x^{c,(\ell-1)}\right)}{f^{P^{C}}\left(x^{c,(\ell-1)}\right)}\right)<0$, as for $\ln \left(\frac{f^{Q^{\mathrm{e}}}\left(x^{c,(\ell-1)}\right)}{f^{P^{\mathrm{c}}}\left(x^{c,(\ell-1)}\right)}\right)>0$ the intersection between the events $\left\{l_{i, j}<-\ln \left(\frac{f^{Q^{\mathrm{e}}}\left(x^{c,(\ell-1)}\right)}{f^{P^{c}}\left(x^{c,(\ell-1)}\right)}\right)\right\}$ and $\left\{l_{i, j}>0\right\}$ is null and 30. boils down to zero. By recalling the distribution of $l_{i, j}$ and employing $\mathrm{Q}(\cdot)$, we have

$$
\begin{aligned}
& p\left(\tilde{l}_{i, j}^{\mathrm{r}(\ell)}<0 \mid \bar{\mu}_{i, j}^{\mathrm{r},(\ell)}=-1, \hat{l}_{i, j}=1\right)= \\
& \frac{\mathrm{Q}\left(\frac{\sigma}{2} \ln \left(\frac{f^{Q^{\mathrm{e}}\left(x^{c,(\ell-1)}\right)}}{f^{P(}\left(x^{c,(\ell-1)}\right)}\right)+\frac{1}{\sigma}\right)-\mathrm{Q}\left(\frac{1}{\sigma}\right)}{p\left(l_{i, j}>0\right)} \cdot \overline{\mathbb{U}}\left(\ln \left(\frac{f^{Q^{e}}\left(x^{\mathrm{c},(\ell-1)}\right)}{f^{P^{C}}\left(x^{c,(\ell-1)}\right)}\right)\right)
\end{aligned}
$$

Similarly, one can compute the following probabilities

$$
\begin{aligned}
& p\left(\tilde{l}_{i, j}^{r,(\ell)}<0 \mid \bar{\mu}_{i, j}^{\mathrm{r},(\ell)}=1, \hat{l}_{i, j}=1\right)= \\
& \frac{\mathrm{Q}\left(\frac{\sigma}{2} \ln \left(\frac{f^{Q^{c}}\left(x^{c,(\ell-1)}\right)}{f^{P e}\left(x^{c,(\ell-1)}\right)}\right)+\frac{1}{\sigma}\right)-\mathrm{Q}\left(\frac{1}{\sigma}\right)}{p\left(l_{i, j}>0\right)} \cdot \overline{\mathbb{U}}\left(\ln \left(\frac{f^{Q^{c}}\left(x^{c,(\ell-1)}\right)}{f^{P^{e}}\left(x^{c,(\ell-1)}\right)}\right)\right) \\
& p\left(\tilde{l}_{i, j}^{\mathrm{r},(\ell)}<0 \mid \bar{\mu}_{i, j}^{r,(\ell)}=0, \hat{l}_{i, j}=1\right)= \\
& \frac{\mathrm{Q}\left(\frac{\sigma}{2} \ln \left(\frac{f^{Q^{\epsilon}}\left(x^{c,(\ell-1)}\right)}{f^{P^{\epsilon}}\left(x^{c,(\ell \ell-1)}\right)}\right)+\frac{1}{\sigma}\right)-\mathrm{Q}\left(\frac{1}{\sigma}\right)}{p\left(l_{i, j}>0\right)} \cdot \overline{\mathbb{U}}\left(\ln \left(\frac{f^{Q^{\epsilon}}\left(x^{c,(\ell-1)}\right)}{f^{P^{\epsilon}}\left(x^{c,(\ell-1)}\right)}\right)\right)
\end{aligned}
$$

By substituting (20)-25) and 27)-(34) into (26), the closed-form expression for $x^{r,(\ell)}$ in (11) is obtained. We remark that in this substitution, $p\left(\hat{l}_{i, j}\right)$ in (26) cancels out with the denominator of (27)-(34).

By substituting $x^{\mathrm{c},(\ell-1)}, \bar{\mu}_{i, j}^{\mathrm{r},(\ell)}$, and $\tilde{l}_{i, j}^{\mathrm{r},(\ell)}$ with $x^{\mathrm{r},(\ell)}, \bar{\mu}_{i, j}^{\mathrm{c},(\ell)}$, and $\tilde{l}_{i, j}^{c,(\ell)}$, and following the same derivation steps as above, Table II and (12) are obtained. This concludes the proof.

\section{APPENDIX B}

ProOF OF PROPOSITION 2

By considering channel adapters, we can assume the transmission of the all-zero codeword in the DE analysis. With 
this assumption and employing (18) and (19), the PDF of the symmetrized LLRs is

$$
p\left(\bar{l}_{i, j} \mid b_{i, j}=0\right)=\sum_{j=0}^{\frac{M}{2}-1} w_{j} \mathrm{G}\left(\bar{l} ; \mu_{j}, \sigma_{j}^{2}\right),
$$

where $w_{j}, \mu_{j}$, and $\sigma_{j}^{2}$ are given in Sec. IV-B The main difference between the DE analysis for BICM and for the bi-AWGN channel in Appendix $\mathrm{A}$ is that the PDF of the LLRs in (35) should be used in the analysis. By employing (35) in (27)-(34) the different terms for 26) are obtained and the expressions in (13) and (14) result for $x^{\mathrm{r},(\ell)}$ and $x^{\mathrm{c},(\ell)}$, respectively. We remark that to run DE the error probability of the VNs should be initialized to $p_{\mathrm{ch}}$. For the BICM channel $p_{\mathrm{ch}}$ can be computed by integrating the tail of the LLR distribution (35), yielding

$$
p_{\mathrm{ch}}=p\left(\bar{l}_{i, j}<0 \mid b_{i, j}=0\right)=\sum_{j=0}^{\frac{M}{2}-1} w_{j} \cdot \mathrm{Q}\left(\frac{\mu_{j}}{\sigma_{j}}\right) .
$$

This concludes the proof.

\section{REFERENCES}

[1] C. Berrou, A. Glavieux, and P. Thitimajshima, "Near shannon limit errorcorrecting coding and decoding: Turbo-codes," in Proc. IEEE Int. Inf. Conf. Commun., vol. 2, Geneva, Switzerland, May 1993, pp. 10641070.

[2] R. G. Gallager, Low-Density Parity-Check Codes. Cambridge, MA, USA: M.I.T. Press, 1963.

[3] K. Cushon, P. Larsson-Edefors, and P. Andrekson, "Low-power 400-Gbps soft-decision LDPC FEC for optical transport networks," IEEE/OSA J. Lightw. Technol., vol. 34, no. 18, pp. 4304-4311, Sep. 2016.

[4] F. Steiner, E. Ben Yacoub, B. Matuz, G. Liva, and A. Graell i Amat, "One and two bit message passing for SC-LDPC codes with higherorder modulation," J. Lightw. Technol., vol. 37, no. 23, pp. 5914-5925, Dec. 2019.

[5] B. P. Smith, A. Farhood, A. Hunt, F. R. Kschischang, and J. Lodge, "Staircase codes: FEC for $100 \mathrm{~Gb} / \mathrm{s}$ OTN," IEEE/OSA J. Lightw. Technol., vol. 30, no. 1, pp. 110-117, Jan. 2012.

[6] Y. Jian, H. D. Pfister, and K. R. Narayanan, "Approaching capacity at high rates with iterative hard-decision decoding," IEEE Trans. Inf. Theory, vol. 63, no. 9, pp. 5752-5773, Sep. 2017.

[7] C. Häger and H. D. Pfister, "Approaching miscorrection-free performance of product codes with anchor decoding," IEEE Trans. Commun., vol. 66, no. 7, pp. 2797-2808, Jul. 2018.

[8] A. Sheikh, A. Graell i Amat, and G. Liva, "Binary message passing decoding of product-like codes," IEEE Trans. Commun., vol. 67, no. 12, pp. 8167-8178, Dec. 2019.

[9] A. Sheikh, A. Graell i Amat, G. Liva, C. Häger, and H. D. Pfister, "On low-complexity decoding of product codes for high-throughput fiberoptic systems," in Proc. IEEE Int. Symp. on Turbo Codes \& Iterative Inf. Proc. (ISTC), Hong Kong, Dec. 2018.

[10] A. Sheikh, A. Graell i Amat, and G. Liva, "Binary message passing decoding of product codes based on generalized minimum distance decoding," in Proc. 53rd Annu. Conf. Inf. Sciences and Systems (CISS), Baltimore, MD, Mar. 2019.

[11] Y. Lei, B. Chen, G. Liga, X. Deng, Z. Cao, J. Li, K. Xu, and A. Alvarado, "Improved decoding of staircase codes: The soft-aided bit-marking (SABM) algorithm," IEEE Trans. Commun., vol. 67, no. 12, pp. 82208232, Dec. 2019.

[12] G. Lechner, T. Pedersen, and G. Kramer, "Analysis and Design of Binary Message Passing Decoders," IEEE Trans. Commun., vol. 60, no. 3, Mar. 2012.

[13] G. Liga, A. Sheikh, and A. Alvarado, "A novel soft-aided bit-marking decoder for product codes," Dublin, Ireland, Sep. 2019.

[14] E. Ben Yacoub, F. Steiner, B. Matuz, and G. Liva, "Protograph-Based LDPC Code Design for Ternary Message Passing Decoding," in Proc. Int. ITG Conf. Syst., Commun. and Coding (SCC), Rostock, Germany, Mar. 2019.
[15] G. Montorsi and S. Benedetto, "High throughput two-stage soft/hard codecs for optical communications," in Proc. IEEE Int. Symp. on Turbo Codes \& Iterative Inf. Proc. (ISTC), Hong Kong, Dec. 2018.

[16] L. M. Zhang and F. R. Kschischang, "Low-complexity soft-decision concatenated LDGM-staircase FEC for high-bit-rate fiber-optic communication," IEEE/OSA J. Lightw. Technol., vol. 35, no. 18, pp. 3991-3999, Sep. 2017.

[17] M. Barakatain and F. R. Kschischang, "Low-complexity concatenated LDPC-staircase codes," IEEE/OSA J. Lightw. Technol., vol. 36, no. 12, pp. 2443-2449, Jun. 2018.

[18] M. Lentmaier and K. S. Zigangirov, "Iterative decoding of generalized low-density parity-check codes," in Proc. IEEE International Symp. on Inform. Theory (ISIT), Boston, USA, Aug. 1998.

[19] R. Tanner, "A recursive approach to low complexity codes," IEEE Trans. Inf. Theory, vol. 27, no. 5, pp. 533-547, Sep. 1981.

[20] C. Häger, H. D. Pfister, A. Graell i Amat, and F. Brännström, "Density evolution for deterministic generalized product codes on the binary erasure channel at high rates," IEEE Trans. Inf. Theory, vol. 63, no. 7 , pp. 4357-4378, Jul. 2017.

[21] E. Zehavi, "8-PSK trellis codes for a rayleigh channel," IEEE Trans. Commun., vol. 40, no. 5, pp. 873-884, May 1992.

[22] G. Caire, G. Taricco, and E. Biglieri, "Bit-interleaved coded modulation," IEEE Trans. Inf. Theory, vol. 44, no. 3, pp. 927-946, May 1998.

[23] G. Böcherer, F. Steiner, and P. Schulte, "Bandwidth efficient and rate-matched low-density parity-check coded modulation," IEEE Trans. Commun., vol. 63, no. 12, pp. 4651-4665, Dec. 2015.

[24] F. Buchali, F. Steiner, G. Böcherer, L. Schmalen, P. Schulte, and W. Idler, "Rate adaptation and reach increase by probabilistically shaped 64 QAM: An experimental demonstration," IEEE/OSA J. Lightw. Technol., vol. 34, no. 7, pp. 1599-1609, Apr. 2016.

[25] F. Gray, "Pulse code communications," U.S. Patent 2632058, Mar. 1953.

[26] A. J. Viterbi, "An intuitive justification and a simplified implementation of the MAP decoder for convolutional codes," IEEE J. Select. Areas in Communications., vol. 16, no. 2, pp. 260-264, Feb. 1998.

[27] J. Hou, P. H. Siegel, L. B. Milstein, and H. D. Pfister, "Capacityapproaching bandwidth-efficient coded modulation schemes based on low-density parity-check codes," IEEE Trans. Inf. Theory, vol. 49, no. 9, pp. 2141-2155, Sep. 2003.

[28] M. Ivanov, C. Häger, F. Brännström, A. Graell i Amat, A. Alvarado, and E. Agrell, "On the information loss of the Max-Log approximation in BICM systems," IEEE Trans. Inf. Theory, vol. 62, no. 6, pp. 3011-3025, Jun. 2016.

[29] J. Max, "Quantizing for minimum distortion," IRE Transactions on Information Theory, vol. 6, no. 1, pp. 7-12, Mar. 1960.

[30] S. Lloyd, "Least squares quantization in PCM," IEEE Trans. Inf. Theory, vol. 28, no. 2, pp. 129-137, Mar. 1982. 